Publisher: Taylor \& Francis \& IAHS

Journal: Hydrological Sciences Journal

DOI: $10.1080 / 02626667.2015 .1022552$

\title{
Analyse multi-échelles de la variabilité spatiale de l'équivalent en eau de la neige (EEN) sur le territoire de l'Est du Canada
}

\author{
Noumonvi Sena, Karem Chokmani, Erwan Gloaguen et Monique Bernier
}

Institut national de la recherche scientifique Centre - Eau Terre Environnement, Québec, Québec G1K 9A9, Canada

noumonvi_yawu.sena@ete.inrs.ca

\begin{abstract}
Résumé La présente étude a pour objectif d'analyser la variabilité spatiale multiéchelles de l'équivalent en eau de la neige (EEN). Dans un premier temps, la variabilité spatiale de l'EEN par rapport à la latitude et à la longitude a été analysée. Des indices locaux ont été utilisés pour caractériser les différentes structures spatiales. Par la suite, les structures spatiales homogènes ont été délimitées à l'aide de l'approche de segmentation spatiale multi-résolutions en utilisant des méta-variables physiographiques. La segmentation a été validée à l'aide du test non paramétrique de Kruskal-Wallis appliqué aux données de l'EEN de chaque paire de zones adjacentes. À l'échelle régionale, la segmentation spatiale a permis d'identifier six zones géographiques différenciées par leur position par rapport aux modes de circulations atmosphériques et la disposition du relief. À l'échelle locale, la segmentation spatiale montre le rôle de la pente, de la courbure, etc. dans la variabilité spatiale du couvert nival.
\end{abstract}

Mots clefs variabilité spatiale ; structure spatiale ; segmentation spatiale ; EEN

Multi-scale analysis of the spatial variability of the water equivalent of snow (EEN) on the eastern territories of Canada

\footnotetext{
Abstract The main objective of this study is to analyze the multi-scale spatial variability of the snow water equivalent (SWE). As a first step, the spatial
} 
variability of SWE, compared to the latitude and the longitude was analyzed.

Local indices have been used to characterize different spatial structures.

Subsequently, the spatial structures were delineated using a spatial segmentation approach (Multi resolutions) on physiographic meta-variables. The segmentation has been validated using the non-parametric test Kruskal-Wallis applied to the SWE data of each pair of geographic areas adjacent. At the regional scale, the spatial segmentation has identified six geographical areas distinguished by their position related to the different modes of atmospheric circulation variability and the disposal of the relief. At the local scale, the spatial segmentation highlighted the role of the slope, curvature etc. in the spatial variability of SWE.

Key words spatial variability; spatial structure; spatial segmentation; SWE

\section{INTRODUCTION}

Le couvert nival occupe une place prépondérante dans le système climatique et dans le cycle hydrologique de l'Est du Canada (Québec et Labrador). Les régions du Québec et du Labrador présentent des accumulations de neige dépassant souvent $300 \mathrm{~mm}$ en termes d'Équivalent en Eau de la Neige (EEN) (Brown 2010). La source principale de données sur l'état du couvert nival est fournie par le réseau de stations nivométriques. Ce réseau a été conçu pour des besoins opérationnels locaux (suivi de l'état du couvert nival, prévisions météorologiques et hydrologiques, etc.), dans les zones habitées et d'intérêts économiques (Braateen et Brown 1998, Brasnett 1999, Brown et al. 2007). Par conséquent, sa densité et sa distribution spatiale ne sont pas optimales dans l'analyse de la variabilité spatiale du couvert nival à l'échelle du territoire. Dans les zones peu couvertes par les stations nivométriques, différentes méthodes d'estimation spatiale telles que les méthodes géostatistiques linéaires (Erxleben et al. 2002), les réseaux de neurones (Evora et al. 2008), les modèles physiques (Turcotte et al. 2006), les modèles hybrides (Goovaerts 2000), etc. sont proposées pour inférer les paramètres physiques du couvert nival (densité, profondeur, EEN). Par ailleurs, la télédétection spatiale (optique, micro-onde passive ou active, etc.) est utilisée comme une source 
alternative de données indirecte sur le couvert nival. Elle est en mesure de fournir des résultats satisfaisants sur la présence ou non de la neige (Brown et Goodison 1996, De Sève et al. 2001, Chokmani et al. 2005, Chokmani et al. 2006). Cependant, les approches par télédétection nécessitent d'être améliorées dans les régions telles que le Québec et le Labrador où les conditions de la neige (couvert nival épais) et le couvert végétal très diversifié (toundra, taïga, forêt boréale, zone agricole) constituent un grand défi pour l'élaboration d'approches efficaces pour estimer les propriétés physiques de la neige et en mesurer sa variabilité spatiale (Brown 2000, Goïta et al. 2003, Turcotte et al. 2006). Cette variabilité spatiale de la neige s'observe à tous les niveaux d'organisation, soit du moléculaire à l'échelle globale. Elle demeure complexe à définir de façon unique et spécifique (Levin 1992, Li. et Reynolds 1995). Deux approches sont privilégiées dans l'étude de la variabilité spatiale des phénomènes biophysiques (Garrigues 2004): l'approche structurelle et l'approche fonctionnelle.

L'approche structurelle se base sur l'analyse des variations de l'intensité du phénomène (par exemple l'EEN) mesurées dans les stations, sans faire appel à aucun processus externe sous-jacent. L'hypothèse est que l'utilisateur dispose d'assez de données précises et sans biais couvrant l'ensemble du territoire. Ces données doivent avoir une densité et une répartition spatiale permettant de caractériser la variabilité spatiale du phénomène en tout point et à l'échelle concernée. Par contre, dans le cas où l'on disposerait de peu de données ou une connaissance partielle du phénomène, l'approche fonctionnelle est plus appropriée. Celle-ci étudie les variations de l'intensité des processus sous-jacents qui contrôlent la variabilité spatiale du phénomène. Ces processus sont entretenus par les facteurs physiographiques (locaux et régionaux) connus en tout point du domaine d'étude. Ces processus sous-jacents génèrent des structures spatiales du phénomène étudié. Ils sont ainsi responsables d'entités spatiales 
homogènes formant un fort contraste avec les structures spatiales des zones environnantes. Les zones de transitions entre les différentes structures spatiales correspondent aux discontinuités spatiales (Adler et al. 2001, Garrigues 2004). Par ailleurs, la structure spatiale dépend de l'échelle spatiale d'observation. En ce qui concerne le couvert nival, la variabilité spatiale se situe à l'échelle régionale entre 10 km et $100 \mathrm{~km}$ et à l'échelle locale entre 100 m et $10 \mathrm{~km}$ (Gray et Male 1981, Marsh 1999). Dans le cas particulier de l'EEN, les mesures dans les stations nivométriques sont réalisées le long d'une ligne de neige de 300 m de longueur (MDDELCC 2008). Par conséquent, dans cette étude, la variabilité spatiale de l'EEN sera étudiée à l'échelle locale de $300 \mathrm{~m} \times 300 \mathrm{~m}$ et à l'échelle régionale de $10 \mathrm{~km} \times 10 \mathrm{~km}$.

Les travaux d'analyse de la variabilité spatiale du couvert nival à l'échelle régionale démontrent que la variabilité spatiale du couvert nival est essentiellement contrôlée par le régime des précipitations (McKay et Gray 1981, Jones et al. 2001). Ce dernier dépend de la position géographique sur le globe et de l'altitude (Brown 2010). De plus, plusieurs travaux mettent en évidence les incidences des téléconnexions (ENSO, El Nino, Pacific North American, etc.) sur le régime de précipitation québécois et canadien. Durant les cycles d'El Niño-Southern Oscillation (ENSO), d'importantes fluctuations pluviométriques (pluie et neige) surviennent le long des régions côtières. Des hivers plus humides marquent les phases froides d'ENSO. Durant la phase chaude d'ENSO, les hivers sont plus secs près des Grands Lacs et légèrement plus humides dans la portion Nord de cette région (Guay et al. 1998). Par ailleurs, la variabilité spatiale du couvert nival est associée aux renforcements des trajectoires des tempêtes des Grands Lacs. Ces derniers sont dynamisés par l'ENSO (Brown et Goodison 1996, Braateen et Brown 1998, Mote et al. 2005, Eichler et Higgins 2006 ). De plus, des liens 
statistiques significatifs sont mis en évidence entre l'ENSO et le ruissellement du printemps dans les bassins hydrographiques du Québec (Rasmussen et al. 1999).

À l'échelle locale, l'accumulation de la neige au sol est contrôlée par les facteurs physiographiques locaux (altitude, proximité des plans d'eau, exposition ou rayonnement solaire, vent, etc.) (Dickison et Daugharty 1977, Plamondon et al. 1984, Hantel et al. 2000, Marty 2008). Généralement, les travaux recensés dans la littérature abordent la variabilité spatiale du couvert nival selon une approche structurelle. Très souvent, la connaissance partielle sur la variabilité spatiale du couvert nival fournie par le réseau nivométrique ne permet pas d'étudier la variabilité spatiale du couvert nival d'une manière optimale à l'aide de telle approche. Par contre, en se basant sur l'approche fonctionnelle, on peut combler ce manque. Cette étude peut mieux aider à comprendre et améliorer la connaissance de la variabilité spatiale du couvert nival. Aussi, elle peut aider à porter un regard critique sur le réseau actuel, à optimiser sa distribution spatiale et contribuer à améliorer les approches d'interpolation.

La présente étude a pour objectif principal d'analyser la variabilité spatiale multi-échelles de l'EEN dans l'Est du Canada et ce, dans le cadre d'une approche fonctionnelle. De manière plus spécifique, l'étude consiste à (a) identifier et caractériser les structures spatiales de l'EEN à l'échelle considérée. La variabilité spatiale de l'EEN sera mise en évidence visuellement en fonction des facteurs physiographiques régionaux (latitude, longitude). Également des indices locaux d'association spatiale seront calculés pour vérifier la présence ou non des structures spatiales en fonction de leur ressemblance; et (b) définir les zones géographiques aux structures spatiales homogènes en termes d'EEN à l'échelle considérée. À ce titre, les zones physiographiques présentant des structures spatiales homogènes seront délimitées à l'aide de l'approche de segmentation spatiale multi-résolutions intégrant les méta- 
variables physiographiques qui contrôlent la variabilité spatiale de l'EEN à chaque échelle. L'intégration des méta-variables sera réalisée à l'aide des statistiques multi variées.

\section{MATERIELS ET METHODES}

\section{Territoire d'étude}

Le territoire d'étude couvre le Québec et le Labrador continental. Il s'étend de la latitude $45^{\circ}$ au $65^{\circ}$ Nord et de la longitude $55^{\circ}$ au $82^{\circ}$ Ouest (Fig. 1). Le relief est relativement modéré et comprend trois ensembles que sont (MDDELCC 2002): les Appalaches, les Basses-terres du fleuve Saint-Laurent et le Bouclier canadien. Ce vaste territoire présente de forts écarts climatiques liés au relief, à la latitude, à sa position septentrionale, à la double exposition aux eaux froides de la Baie d'Hudson et aux courants océaniques froids le long de la côte du Labrador. Quatre principaux climats marquent le territoire. Ce sont : le climat continental humide (au Sud du $50^{\mathrm{e}}$ degré Nord), le climat subarctique (entre les $50^{\mathrm{e}}$ et $58^{\mathrm{e}}$ degrés), le climat arctique (plus au Nord) et le climat maritime à l'Est (MRN. 2013). Cet ensemble de climats moins favorable au fur et à mesure que l'on remonte vers le Nord, détermine la distribution de la végétation. Les principales zones de végétation comprennent la zone de végétation tempérée nordique, la zone de végétation boréale et la zone de végétation arctique (MRN. 2013) (Fig. 1). Ces trois zones sont constituées de sous-zones en fonction de la physionomie de la végétation.

Données nivales Le réseau nivométrique du territoire d'étude est constitué de 426 stations et les stations qui ont 10 ans et plus ont été retenues dans cette étude. Cette période d'observation constitue la durée minimale pour tenir compte des évènements atmosphériques et océaniques cycliques (cycle solaire, El NiñoSouthern Oscillation, Nina, etc.) susceptibles d'influencer l'accumulation de neige 
(Rasmussen et al. 1999, Sobolowski et Frei 2007). Pour cette étude, on se base sur l'hypothèse que le phénomène neige est stationnaire durant la période d'observation. Au total, 367 stations de 10 ans et plus sont retenues comprenant 193 stations du Ministère du Développement Durable, Environnement et Lutte contre les Changements Climatiques (MDDELCC), 19 stations de RioTinto, 76 stations d'Hydro-Québec et les données historiques de 79 stations fournies par Environnement Canada.

Selon les protocoles d'échantillonnage des paramètres physiques de la neige, les mesures de la station sont représentatives de la variabilité des conditions de neige dans un voisinage restreint de la station (MSC. 2004, MDDELCC 2008). La station est constituée de 10 repères d'échantillonnage répartis uniformément le long d'une ligne de neige de $300 \mathrm{~m}$ de longueur et située en milieu forestier afin de la protéger des vents violents, mais bien dégagée. Dans chaque repère d'échantillonnage, la hauteur de la neige ainsi que l'EEN sont relevés par la moyenne des carottages aux 10 points. À chaque repère, si la hauteur de la neige est supérieure à $25 \mathrm{~cm}$, le poids de la carotte est déterminé. L'EEN est obtenu par soustraction du poids du carottier vide de cette mesure. Par contre, si la hauteur de neige est inférieure à $25 \mathrm{~cm}$ à au moins un repère, l'EEN est obtenu après avoir soustrait le poids du seau vide de la mesure cumulative des carottes de tous les repères. La densité de la neige dérive de la hauteur et de l'équivalent en eau. Les mesures sont bihebdomadaires et sont plus fréquentes de janvier à mai. Pour chaque station, le maximum annuel de l'EEN a été calculé. Le maximum annuel de l'EEN estime le plafond des taux de fonte de neige (Perrier 1968). Sur le plan opérationnel, le maximum moyen annuel de l'EEN conditionne les ressources hydriques maximales à la fonte printanière. Par la suite, les variables statistiques de tendance centrale (moyenne des maxima annuels) et de dispersion (écart-type, intervalle interquartile, Q50) ont été calculées pour toutes les stations considérées. 
Processus sous-jacents à la variabilité spatiale de l'EEN À l'échelle régionale, les processus sous-jacents à la variabilité spatiale de l'EEN sont abordés par des travaux récents à travers les effets météorologiques dynamiques. Ces effets météorologiques sont principalement les écarts de déviation dus aux forces de Coriolis, aux ondes stationnaires dans l'atmosphère, les flux des vents le long des montagnes, les changements de températures latitudinales et les sources d'humidité régionaux (océans) (Jones et al. 2001). Les circulations atmosphériques à grande échelle sont régies par ces éléments météorologiques qui conditionnent les types de précipitations. Dans le cas particulier de la variabilité spatiale du couvert nival, les grandes étendues d'eau (Grands Lacs, océan) créent un microclimat qui alimente des chutes de neige sur les côtes méridionales et orientales du Québec continental: c'est la Snow Belt (Byrd 2000). L'air froid de passage sur les eaux chaudes se charge d'humidité formant des nuages convectifs très intenses qui donnent de la neige au-dessus des terres. C'est de la neige en abondance qui tombe en quelques heures le long de minces corridors (Byrd 2000). Aussi, le ciel est souvent nuageux en hiver tant que la température de l'air est plus froide que la température des eaux de la mer et les grandes étendues d'eau ne sont pas gelées. Au Sud du Québec, la forme typique d'accumulation nivale qui fait la spécificité du couvert nival est due à l'influence des Grands Lacs. Dans le cas des océans, l'humidité océanique diminue vers l'intérieur des terres. C'est l'effet de la continentalité. La longitude est un facteur de répartition des types de précipitations (Oetlli et Camberlin 2005). Plusieurs études démontrent la variabilité spatiale du couvert nival en fonction des oscillations atmosphériques et de la longitude au Nord-Est de l'Amérique du Nord (Brown et Goodison 1996, Brown 2010). Par conséquent, la longitude et la distance à l'océan sont des facteurs physiographiques importants dans l'analyse de la variabilité spatiale du couvert nival. Par ailleurs, à chaque latitude correspondent des types de climats qui diffèrent du Sud au Nord. Généralement, le Nord du Québec est caractérisé par de forts gradients spatiaux dans la durée de la couverture de neige allant de 8 mois (octobre-juin) et de 4 mois (décembre à avril) pour le Sud du Québec (Brown 2010). La température moyenne hivernale est de -13.25 C à Fort-Chimo (Lat. 58 06'; long. 68 24') contre 8.7 C à Wright (Lat. 46 2'; long. 76 1') (MDDELCC 2001). 
Par ce découpage, la latitude est considérée comme un facteur à saisonnalité marqué (Oetlli et Camberlin 2005). Cependant, dans les zones climatiques, les rythmes thermiques et pluviométriques en milieu montagnard sont proches de ceux des plaines voisines, mais les températures sont plus basses et les précipitations (pluie, neige) augmentent au moins jusqu'à une altitude qualifiée d'optimale (Daly et al. 1994, Beltrando et Chémary 1995). Des liens sont établis à l'échelle des vallées entre les précipitations neigeuses et l'élévation (Kronholm et Birkeland 2007). Pour cela, à l'échelle régionale, la longitude, la latitude, l'altitude et la distance à l'océan sont les facteurs physiographiques retenus. Ils sont extraits à partir des données numériques d'élévation du Canada à la résolution de $10 \mathrm{~km} \times 10 \mathrm{~km}$ (RNC 2000).

À l'échelle locale, les travaux recensés dans la littérature montrent que la pente, l'orientation, les courbures de versants, la distance aux plans d'eau et la hauteur des végétaux conditionnent les processus sous-jacents à la redistribution de la neige après précipitation et de son maintien au sol (Gray et Male 1981, Jones et al. 2001). Selon la pente, sous l'action du vent, de l'état du site de dépôt et de l'effet de gravité, les particules de neige s'organisent pour former un couvert nival spécifique. Par ailleurs, le couvert nival dure plus longtemps dans les zones peu exposées (ubacs) que dans les zones plus exposées (adrets). Pour cela, l'emplacement des stations nivométriques tient compte de l'orientation et de l'exposition aux radiations solaires (Watson et Anderson 2006). Aussi, l'accumulation de la neige n'est pas identique sur les différentes courbures (concavité, convexité et convexo-concavité) issues de processus géomorphologique le long des versants. En effet, la neige qui arrive au sol s'organise selon la gravité, son poids, la rugosité et la forme du site de dépôt pour présenter un manteau neigeux spécifique. Pour cela, les interfluves, les sommets découverts contrastent avec les fortes accumulations des combes à neige (Fillon et Payette 1976). 
D’une manière générale, les formes d'un site à un autre interviennent dans l'estimation précise de l'équivalent en eau de la neige à l'échelle locale (Carroll et Cressie 1997). De même, les courbures des versants sont relativement importantes dans l'organisation des phénomènes météorologiques à l'échelle locale, car elles permettent de modéliser les effets de la topographie sur l'organisation des masses d'air (Fillon et Payette 1976, Lhotellier 2005). L'accumulation de la neige diffère selon la proximité des plans d'eau, le type de vent et la température de l'air (McKay et Gray 1981). En effet, dans les latitudes nordiques, la température du microclimat réorganise l'état physique des particules de neige. Les plans d'eau créent un microclimat qui alimente des bourrasques de neige et augmentent l'action du vent. La température et l'humidité de l'air se modifient au voisinage des plans d'eau pour donner de la neige humide lourde moins susceptible d'être transportée par le vent (McKay et Gray 1981, Palacios et SánchezColomer 1997). Aussi, les plans d'eau interviennent dans la dynamique de l'interface végétation-vent ou obstacle-vent. Selon la hauteur du couvert végétal, le vent, principal agent de transport des particules de neige a des capacités de mobilisation et de dépôts divers. Cependant, sa mesure et sa modélisation sont complexes en tout point de l'espace (Swanson 1970). Pour y parvenir, dans cette étude son action est comparée à la proportion de rugosité issue de l'interface vent-couvert végétal ou vent-obstacle. Les hauteurs des végétaux n'étant pas les mêmes, alors les interfaces vent-couvert végétal présentent différentes dynamiques d'interception des particules de neige. Par conséquent, la distribution de la neige n'est pas la même dans les secteurs forestiers, les prairies, la toundra ou sur les surfaces exploitées (sols agricoles ou friches). Au regard de tout ceci, les facteurs physiographiques retenus à l'échelle locale sont: la pente, l'orientation, les courbures des versants, la radiation solaire globale d'hiver et la distance aux plans d'eau. Ils sont extraits à partir des données numériques d'élévation 
du Canada à la résolution de $300 \mathrm{~m} \times 300 \mathrm{~m}$ (RNC 2000). Quant à l'action de l'interface vent-couvert végétal, elle est modélisée à travers les hauteurs des différents types de végétation fournies par l'Inventaire Forestier National du Canada (IFNC. 2004). Pour ce faire, les différents types de végétation sont extraits à partir de la carte d'occupation du sol produite par le Centre Canadien de Télédétection (CCT 1999) et rééchantillonnés à la résolution spatiale de $300 \mathrm{~m} \times 300 \mathrm{~m}$.

\section{Approche méthodologique}

Caractérisation des structures spatiales La modélisation de la variabilité spatiale du couvert nival passe par une description complète des structures spatiales (Kronholm et Birkeland 2007). À cet effet, plusieurs travaux recensés dans la littérature ont analysé la variabilité spatiale des phénomènes climatiques (Fortin et al. 1982), de la production agricole (Ben Arfa et al. 2008, Caillault et al. 2009) et de la démographie (Morency 2006). En ce qui concerne le couvert nival, la structure de la variabilité spatiale de la hauteur de neige est décrite par la régression multiple les débits à l'exutoire des bassins versants en fonction du volume de neige et la période de fonte ultérieure (Dyer 2008). L'analyse variographique est également utilisée pour décrire la variabilité des mesures d'enneigements et en définir les structures spatiales au sol (Kronholm et Birkeland 2007). Dans ce travail, il s'agira de mettre en évidence, de décrire et enfin d'identifier les limites objectives des différentes structures spatiales. Pour y parvenir, une phase de mise en évidence visuelle et quantitative des structures spatiales est menée. Les structures spatiales sont mises en évidence visuellement en fonction de la position géographique et quantitativement à l'aide de l'Indice d'Association Spatiale. Dans la phase de mise en évidence visuelle, les structures spatiales potentielles de la variabilité de l'EEN sont identifiées à l'échelle régionale à l'aide des figures portant en ordonnée les données les maxima annuels moyens de l'EEN des stations et en abscisse la latitude et la longitude. Par la suite, dans l'analyse quantitative, les structures spatiales ont été mises en évidence par la mesure du degré de ressemblance d'une structure spatiale avec ses voisins (Anselin 1995). Cette méthode de calcul des indicateurs locaux d'association 
spatiale est fonction de la distance entre les observations et propose des regroupements spatiaux locaux des structures selon leur ressemblance ou dissemblance. Les observations extrêmes, atypiques sont mises en évidence. L'Indice Local d'Association spatiale (LISA en anglais) est de la forme:

$$
I_{i}=\frac{x_{i}-\bar{X}}{S_{i}^{2}} \sum_{j=1, j \neq i}^{n} w_{i, j}\left(x_{j}-\bar{X}\right)
$$

Où $x_{i}$ est la variable à l'étude, $n$ le nombre d'observations, $\bar{X}$ la moyenne de la variable $x_{i} ; w_{i, j}$ le poids spatial entre les variables $i, j$ et

$$
S_{i}^{2}=\frac{\sum_{j=1, j \neq i}^{n} w_{i, j}}{n-1}-\overline{X^{2}}
$$

Cet indice local d'autocorrélation spatiale montre l'association et la dépendance entre les observations aux différents endroits de l'espace. Il identifie si les structures sont plus ou moins proches les unes des autres (Laffitte et Marc 2010). Mais, cet indice est limité par le choix de la matrice de voisinage et ne permet pas de modéliser ni la forme ni les limites explicites des structures spatiales.

Segmentation spatiale La segmentation spatiale permet de découper l'espace en structures homogènes et distinctes par un ou plusieurs critères significatifs, pertinents, mesurables et accessibles (Drăguţ et al. 2010). Ces structures spatiales homogènes se caractérisent comme un ensemble de pixels ayant des variances et des moyennes communes (intensité, texture, etc.) qui les différencient des pixels des régions voisines. En télédétection, la segmentation spatiale est utilisée pour découper les images radars et/ou optiques dans plusieurs domaines pour faire la classification. Il existe deux approches de segmentation spatiale à savoir l'approche descendante et l'approche ascendante. L'approche descendante part de l'image entière pour la segmenter en petites régions homogènes en référence à des attributs spectraux ou texturaux. L'image est segmentée selon le principe du diagramme de Voronoï. Dans le cas de l'approche ascendante, le pixel est 
sélectionné soit par la méthode dite de bassin versant, soit par un semi de pixels tirés au hasard sur la base de la distance spectrale inférieure à un seuil fixé ou soit au moyen d'un filtre moyen pour lisser l'image et donner naissance à des régions (Nagao et Matsuyama 1979). Dans cette étude, les processus sous-jacents connus en tout point du territoire d'étude sont représentés par les pixels de l'image sous forme de grille régulière. Pour cela, l'approche ascendante de croissance de région a été choisie. Les pixels sont progressivement agrégés à leurs voisins pour créer des objets de plus en plus grands en respectant le seuil d'hétérogénéité prédéfini. Des objets adjacents de taille et de forme similaires bien repartis dans l'image sont produits. Les paramètres définis par l'utilisateur tels que la couleur (valeur spectrale des objets) et la forme (texture) sont pondérés pour former le seuil d'hétérogénéité. La segmentation spatiale a été effectuée par le logiciel eCognition Définiens via la segmentation multi-résolutions qui intègre l'analyse multi-échelles de l'image (Baatz et Schäpe 2001). La segmentation multirésolutions permet de créer différents niveaux qui s'empilent les uns sur les autres, en morcelant ou fusionnant les objets issus de la segmentation initiale. Chaque niveau de segmentation s'emboite avec le précédent et le suivant.

L'objectif de ce travail est d'identifier les limites objectives entre les structures spatiales homogènes à l'échelle locale et régionale. Pour y parvenir, la démarche méthodologique de la segmentation spatiale adoptée se compose de trois principales étapes (Fig. 2). À la première étape, au sein d'un projet sont constituées des métavariables utilisées pour segmenter l'espace géographique. À la seconde étape, l'algorithme multi- résolutions est paramétré en fixant les seuils de la valeur spectrale (couleur), de la forme (texture) et les facteurs d'échelle. Les poids des méta-variables déterminés par les statistiques multivariées sont intégrés. La dernière étape de la segmentation spatiale est la validation des résultats par le test non paramétrique Kruskal-Wallis.

Construction des méta-variables Dans la construction des méta-variables, les facteurs physiographiques responsables des processus sous-jacents sont calculés par la méthode 
de géomorphométrie générale avec le logiciel ArcGIS à l'échelle régionale et locale. Souvent, ces facteurs physiographiques sont optimisés pour expliquer les variables cibles à l'aide des statistiques multivariées telles que l'analyse en composante principale (ACP) ou l'analyse canonique de corrélation (ACC). Dans ce travail, il s'agit d'optimiser les facteurs physiographiques par une combinaison linéaire des vecteurs maximisant la corrélation entre les variables canoniques. Dans de pareils cas, l'Analyse Canonique de Corrélation (ACC) est adaptée, car elle permet de caractériser les relations linéaires pouvant exister entre deux ensembles de variables aléatoires (Croux et Dehon 2003, Baccini et Besse 2004). Aussi, elle permet la détermination des paires de combinaisons linéaires de chaque ensemble de variables, nommées les variables canoniques, de manière telle que la corrélation entre les variables canoniques d'une paire est maximisée et la corrélation entre les variables de différentes paires est nulle. On obtient ainsi un ensemble de variables canoniques pour les deux ensembles de variables aléatoires (Haché et al. 2002).

Étant donné les ensembles de facteurs physiographiques retenus, $X$ (échelle régionale et locale) et l'ensemble de variables du maximum annuel moyen de l'EEN, $Y$ (statistique du maximum annuel), l'ACC a pour but de relier les deux ensembles en utilisant des vecteurs de variables canoniques : $U$ pour les variables physiographiques et $V$ pour les variables de l'EEN. Les variables $U$ et $V$ sont les combinaisons linéaires de $X$ et $Y$. Les coefficients des combinaisons linéaires sont estimés par la maximisation de la corrélation entre les variables aléatoires $U$ et $V$. Le coefficient de corrélation entre chaque paire d'éléments de vecteur, soit $U$ ou $V$ est nul. Les variables canoniques ( $U$ et $V)$ sont données par le système d'équations linéaires suivant :

$$
\begin{aligned}
& U=\alpha_{1} X_{1}+\ldots+\alpha_{q} X_{q}=\alpha X \\
& V=\beta_{1} Y_{1}+\ldots+\beta_{r} Y_{r}=\beta Y
\end{aligned}
$$


Avec $r \leq q$ représentant respectivement l'ensemble des $r$ variables expliquées (l'occurrence les variables du maximum annuel de l'EEN) et l'ensemble $q$ des variables dites explicatives (en l'occurrence les facteurs physiographiques). Les vecteurs des paramètres $\alpha$ et $\beta$ sont estimés sous les contraintes suivantes:

(1) Maximiser $\operatorname{Corr}\left(U_{i}, V_{i}\right)=\lambda_{i}$ avec $i=1, \ldots, p$ ( $p$ le rang de la matrice de variancecovariance des $X$ et $Y$ ) et $\lambda$ le coefficient canonique de corrélation;

(2) $\operatorname{Corr}\left(U_{i}, V_{i}\right)=0$ avec $i \neq j$

(3) $\operatorname{var}\left(U_{i}\right)=\operatorname{var}\left(V_{j}\right)$ avec $i=1, \ldots, p$

Les variables sont normalisées pour avoir une distribution gaussienne par la technique de normalisation Boxcox et sont centrées pour avoir des valeurs représentatives de leur moyenne selon les équations (4) et (5):

$$
\begin{aligned}
& X_{T(k)}=\operatorname{boxcox}\left(X_{k}\right) \\
& X_{k}^{\prime}=\left(X_{T(k)}-\overline{X_{T(k)}}\right)
\end{aligned}
$$

où $k$ prend les valeurs des facteurs physiographiques selon l'échelle considérée.

Le poids de la méta-variable $U_{i}$ est le rapport du coefficient canonique de corrélation entre la méta-variable physiographique $\left(U_{i}\right)$ et la variable canonique d'EEN $\left(V_{i}\right)$ au carré sur la somme totale des coefficients canoniques de corrélation au carré. L'équation du poids est de la forme :

$$
P_{U_{i}}=\lambda_{i}^{2} \frac{1}{\sum_{i=1}^{k}\left(\lambda_{i}\right)^{2}}
$$

Les méta-variables de l'EEN ( $V$ ) sont ponctuelles et pas connues en tout point de l'espace. Par contre, les méta-variables physiographiques $(U)$ optimisées par la variable de l'EEN sont connues en tout point de l'espace. Elles représentent les processus sous- 
jacents qui conditionnent la variabilité spatiale de l'EEN selon l'échelle d'observation. Par conséquent, seules les méta-variables $(U)$ sont intégrées dans l'approche de segmentation multi-résolutions.

\section{Algorithme de segmentation multi-résolutions La segmentation multi-résolutions} regroupe les pixels sur la base des critères d'homogénéité et de l'échelle. Le critère d'homogénéité $\left(S_{f}\right)$ se compose de deux paramètres que sont : l'hétérogénéité de la couleur $\left(h_{\text {couleur }}\right)$ et l'hétérogénéité de la forme $\left(h_{\text {forme }}\right)$. Sa formule est :

$$
S_{f}=W \times h_{\text {couleur }}+(1-W) \times h_{\text {forme }}
$$

où $0 \leq W \leq 1$ est le poids défini pour la valeur forme ou la couleur. Les poids de la forme et de la couleur sont complémentaires et leur somme est égale à 1. La segmentation spatiale exige un minimum de poids de l'hétérogénéité de la couleur et de l'hétérogénéité de la forme (Alban 2005). En effet, plus l'hétérogénéité de la forme est pondérée moins l'hétérogénéité de la couleur influence la segmentation et vice versa. Par ailleurs, pour l'hétérogénéité de la forme, les valeurs de compacité et de lissage influent également sur la création de l'objet. Une forte pondération du facteur de compacité se traduit par des objets plus compacts et une faible pondération du lissage favorise les formes plus linéaires. Tout comme l'hétérogénéité de la couleur et l'hétérogénéité de la forme, les poids de la compacité et du lissage sont complémentaires et leur somme est égale à 1 .

La segmentation spatiale exige également la prise en compte du critère d'échelle. Ce critère limite l'hétérogénéité acceptable au sein des objets. Il fournit les facteurs d'échelle à partir desquels les objets regroupés déterminent des régions aux structures homogènes. Dans ce travail, l'outil d'ESP (Estimation of Scale Parameters) est utilisé (Drăguţ et al. 2010). L'ESP segmente l'image avec des incréments fixes de paramètres d'échelle et calcule la variance locale de l'hétérogénéité des objets sur l'image en moyennant les variances des objets pour chaque niveau d'objet obtenu par la 
segmentation. La croissance de la variance locale est proportionnelle au paramètre d'échelle à mesure que l'homogénéité des objets de la scène augmente. Le graphique de la variance locale (VL) permet d'évaluer les paramètres d'échelle appropriés (Drăguţ et Eisank 2011). Pour évaluer la dynamique de la variance locale, le graphique du taux de changement de la variance locale (TC) d'un niveau d'objet à un autre est ajouté. Les pics dans le graphique TC-VL correspondent aux changements de structures. Ils indiquent les facteurs d'échelle appropriés auxquels l'image peut-être segmentée par rapport à la variance locale des objets. Entre deux pics, les facteurs d'échelles secondaires n'apportent que de légères modifications dans la segmentation (Drăguţ et al. 2010).

Validation Il n’y a pas de données disponibles, permettant de vérifier si les limites établies par les segmentations spatiales décrites plus haut ont un sens réel sur la variabilité spatiale. Le seul moyen disponible est de vérifier si les observations de l'EEN au niveau des stations situées à l'intérieur d'une zone géographique aux structures spatiales homogènes sont significativement différentes de celles des groupes de stations dans les zones géographiques contiguës à la zone en question. Pour ce faire, le test de comparaison statistique non paramétrique de Kruskal-Wallis est appliqué. Ce test est une alternative à l'analyse de variance des échantillons. Le test de KruskalWallis est adapté aux échantillons de taille faible et compare les rangs des $x_{i}$ (données de l'EEN) dans leur liste ordonnée et non pas leur valeur (Poinsot 2004, Vinatier 2008). La taille minimale des échantillons pour comparer deux échantillons sans estimer la moyenne et la variance varie entre 3 et 5 (Poinsot 2004, Dodge 2007). Dans cette étude, seules les zones géographiques comportant quatre stations et plus ont été retenues. Ce choix est une moyenne des différentes limites proposées par la littérature. Le seuil de 
signification (ou risque d'erreur) de 5\% est retenu pour démontrer qu'au moins un groupe de stations diffère significativement des autres groupes de stations des zones adjacentes.

\section{RESULTATS ET DISCUSSION}

\section{Caractérisation des structures de la variabilité spatiale de l'EEN}

La Fig. 3(a) et (b) montre en abscisse les variables physiographiques (latitude et longitude) et en ordonnée le maximum annuel moyen de l'EEN. Quatre sections de structures de la variabilité spatiale de l'EEN par rapport à la longitude peuvent être identifiées visuellement (Fig. 3(a)). Entre les longitudes 57.18 et 65.03 Ouest (Fig. 3(a), Section I), il y a une étendue des valeurs du maximum annuel de l'EEN de 101 à 398 $\mathrm{mm}$. Cette section correspond à l'extrême Est du territoire d'étude. La forte moyenne du maximum annuel de l'EEN (291 mm) est liée aux actions combinées de l'altitude et de l'influence de l'océan. En effet, les précipitations abondantes dans les zones maritimes sur les reliefs élevés des monts (Jacques Cartier 1268 m, Kapatahkatnahiu 623 m) expliquent la croissance de la moyenne d'EEN en allant de l'Est vers l'Ouest. La Section II (long. 65.04 à 72.84 Ouest) correspond au climat continental humide du Sud et la partie centrale du Bouclier canadien. La moyenne (264 mm) et le maximum (515 mm) de l'EEN peuvent s'expliquer par la présence des Monts situés au contact des provinces géologiques du Bouclier canadien (Lac-Supérieur, Churchill, Greenville). L'action de l'altitude est un facteur explicatif à cette longitude, car l'influence des vents océaniques est moins importante qu'à la section I. La variabilité $(76 \mathrm{~mm})$ peut être due en partie aux zones habitées où l'échantillonnage est plus dense. La Section III (long. 72.84 à 75.56 Ouest) s'ouvre sur les piémonts des plateaux et des monts du Bouclier canadien. Elle a une moyenne de $203 \mathrm{~mm}$ avec une tendance à la baisse et une variance de $49 \mathrm{~mm}$. Ceci peut s'expliquer par l'exposition de cette partie Ouest du Bouclier canadien aux vents humides des baies de Hudson et de James. Au Sud de cette section, c'est le domaine du climat continental humide, tandis que le centre et le Nord correspondent respectivement aux climats subarctique et arctique. La Section IV (long. 75.56 à 79.83 Ouest) englobe les basses terres de l'Ouest sous l'influence des vents humides des baies (Hudson et James). Le maximum annuel moyen de l'EEN est plus 
élevé que dans la section III. Cependant, la section IV présente une faible moyenne d'EEN (170 mm) et un faible écart-type $(35 \mathrm{~mm})$ par rapport aux autres sections.

Du Nord au Sud, visuellement, on peut distinguer cinq structures spatiales de la variabilité spatiale de l'EEN (Fig. 3b). La Section I (lat. 45.01 à 47.45N) correspond aux zones habitées où la densité des stations de mesure est plus élevée par rapport au Nord. Dans ce secteur, le climat continental humide avec ses forêts mixtes et le relief élevé des Laurentides tel que les Monts de la Tuque expliquent les fortes valeurs du maximum annuel de l'EEN (515 mm). De latitude 47.45 à $48.85 \mathrm{~N}$, se localise la Section II qui se différencie de la section I par sa tendance stationnaire avec un maximum de $392 \mathrm{~mm}$ et un écart-type de $47 \mathrm{~mm}$. C'est un domaine forestier de feuillus et de conifères, cependant le relief est plus accidenté par rapport à la Section I. La Section III (lat. 48.85 à $52.17 \mathrm{~N}$ ) avec une moyenne de $260 \mathrm{~mm}$ et un écart-type de $50 \mathrm{~mm}$ correspond à la partie méridionale du Bouclier canadien et les basses terres de l'Ouest. En effet, en remontant le bouclier, l'altitude augmente et le relief devient plus accidenté. Cependant, cette section est moins échantillonnée dans sa partie Ouest que dans la Section II. La Section IV (lat. 52.17 à $55.03 N$ ) correspond aux domaines des principaux monts du bouclier et à la végétation nordique. La forte variabilité du maximum annuel de l'EEN peut être due à la présence des monts du bouclier et de la végétation forestière boréale et humide. Au-delà de la latitude 55.03N (Section V), c'est le domaine arctique avec une tendance à la basse et parfois stationnaire. C'est un territoire ouvert où la température très basse, pouvant atteindre $-40 \mathrm{C}$, associée aux forts vents, donne de la neige difficilement mesurée dâns les rares stations nivométriques. En somme, quatre et cinq grandes structures potentielles de la variabilité spatiale de l'EEN respectivement selon la longitude et la latitude peuvent être identifiées visuellement. Elles se rapprochent des classes de climat avec des variances liées aux effets combinés du relief et du climat. De manière quantitative, l'Indice d'Association Spatiale Locale (LISA : local index of spatial association) vient confirmer le constant visuel et démontre l'existence des structures spatiales de l'EEN. Cet indice se base sur l'hypothèse que les valeurs de l'EEN en un point sont fonction des valeurs dans le voisinage. Cet indice identifie trois structures spatiales différentes (Fig. 4). Une structure aux faibles valeurs d'EEN au Sud du domaine d'étude et une structure aux valeurs élevées de l'EEN sur le plateau du bouclier. Elles sont séparées par la structure aux valeurs intermédiaires d'EEN que les statistiques spatiales de l'LISA ne permettent pas de classer dans l'une ou l'autre structure. Des structures de groupe de valeurs extrêmes (fortes ou faibles) 
sont également identifiées. Ces deux méthodes de mise en évidence visuelle et quantitative montrent que l'EEN n'est pas stationnaire aussi bien au niveau de sa moyenne que de sa variance dans l'espace. Cependant, elles ne permettent pas de délimiter explicitement les limites spatiales objectives de chaque structure. Ceci justifie le recours à la segmentation spatiale que nous proposons à la section suivante

\section{Segmentation spatiale}

Echelle régionale - Analyse exploratoire des données La Fig.5 montre les histogrammes, les diagrammes de dispersion ainsi que les coefficients de corrélations entre les variables statistiques du maximum annuel de l'EEN et les variables physiographiques à l'échelle régionale. L'ensemble des histogrammes des variables est asymétrique ce qui impose une transformation normale préalable à l'application de l'analyse canonique de corrélation. La moyenne, la médiane et l'interquartile du maximum annuel de l'EEN ont des distributions asymétriques à gauche tandis que la distribution de l'écart-type est symétrique. La latitude présente une distribution presque bimodale en raison de la forte présence de stations au Sud du territoire. La longitude a une distribution asymétrique à droite du fait de la forte présence de stations à l'Est du territoire. L'altitude et la distance à l'océan ont une distribution presque normale. Le coefficient de corrélation le plus élevé s'observe entre l'altitude et la moyenne du maximum annuel de l'EEN. En effet, les précipitations de neige croissent avec l'altitude où la température est plus basse (Beltrando et Chémary 1995). À l'inverse, le coefficient de corrélation $(r=-0,47)$ entre la longitude et la moyenne du maximum annuel de l'EEN montre qu'avec l'effet de continentalité, à mesure que l'on s'enfonce dans les terres, le maximum annuel moyen de l'EEN baisse. De même, la corrélation négative entre la distance à l'océan et le maximum annuel moyen de l'EEN démontre la baisse de l'influence des masses d'air humide au fur et à mesure que l'on s'éloigne des côtes. En hiver, les vents d'Ouest (dépression d'Islande), chargés d'humidité, donnent plus de neige dans la partie est du territoire d'étude que vers l'intérieur des terres (Leduc et Gervais 1985). La corrélation entre la latitude et la moyenne du maximum annuel de l'EEN est relativement faible, mais significative $(p<0.05)$. Comme il a été mentionné plus haut, dans les hautes latitudes, la neige tombe sous une forme très différente de celle des latitudes 
moyennes. Ce sont de fins cristaux en forme de colonne ou de plaques épaisses. L'air se remplit de fines paillettes qui flottent longuement avant d'attendre le sol. Ce sont des chutes horizontales rarement enregistrées par les stations nivométriques ce qui fausse souvent les mesures (Corbel 1958). A ceci s'ajoute la faible présence de stations dans ces latitudes.

Méta-variables à l'échelle régionale La méta-variable $U_{i}$ présente une gamme de patrons spatiaux correspondant aux patrons spatiaux de la variable altitude (Fig. 6(a)). La variable altitude est la plus corrélée avec la moyenne du maximum annuel de l'EEN ( $r=0,64)$ (Fig. 5). La méta-variable $U_{1}$ a le point $\left(P_{U 1}\right)$ le plus élevé $(0,69)$. La métavariable $U_{2}$ a un poids $P_{U 2}$ de 0,26 et présente moins de patrons spatiaux par rapport à $U_{1}$ (Fig. 6(b)). La méta-variable $U_{2}$ présente une gamme de variation similaire aux patrons spatiaux de la variable de la longitude. Les méta-variables $U_{3}$ et $U_{4}$ ont des poids respectifs de 0,03 et 0,001 . Elles présentent peu de patrons spatiaux par rapport aux deux premières méta-variables (Fig. 6(c) et (d)).

Segmentation spatiale à l'échelle régionale L'ESP a été définie dans la section précédente comme l'outil de détermination des facteurs d'échelle auxquels la segmentation spatiale peut être effectuée. Ainsi, aux différents facteurs d'échelle identifiés par des carrés (Fig. 7), il est à constater qu'en augmentant le facteur d'échelle, le nombre de zones géographiques présumées ayant des structures spatiales homogènes décroit de 131 zones aux facteurs d'échelle 4 à 6 zones au facteur d'échelle 30. Seul le résultat de la segmentation avec le facteur d'échelle 30 répondant au test de validation est présenté et discuté.

Les six zones géographiques se différencient par leurs caractéristiques physiographiques (Fig. 8). La zone A englobe les plaines des baies Hudson et James, et touche à l'est les piémonts des hauts reliefs du Bouclier canadien. C'est un domaine de 
plaine sous la forte influence des vents polaires avec une moyenne d'EEN estimée à $183 \mathrm{~mm}$. La Zone B, dans l'axe Nord-Est à Sud-Ouest, regroupe une grande partie du relief moyen (monts Tichégami, 776 m, et Otish, $1128 \mathrm{~m}$ ) située dans la zone de contact des unités géologiques du Bouclier canadien (Lac Supérieur, Greenville et Churchill). L'effet de l'altitude explique la moyenne de 260 mm d'EEN observée sur l'ensemble de cette zone. La partie Ouest de la Zone B inclut les piémonts $(300 \mathrm{~m})$ du Bouclier canadien menant aux plaines des baies de Hudson et James. Elle est sous l'influence des vents polaires du Nord. La Zone C localisée au Nord du territoire d'étude a un maximum annuel moyen d'EEN de $244 \mathrm{~mm}$. Elle inclut les reliefs accidentés des hautes terres du Nord-Ouest (Cratère des Pingualuit) et du Nord-Est du Labrador (Mont Jacques-Rousseau, $1261 \mathrm{~m})$. Elle est sous l'influence des vents froids de l'Arctique. La Zone D s'étire, du Nord au Sud, le long de la rive Nord du fleuve Saint-Laurent. Elle inclut les hautes terres du Nord et Nord-Est du Labrador et la partie orientale du Bouclier canadien. L'influence océanique très forte sur le relief littoral explique la forte moyenne du maximum annuel d'EEN (320 mm). Au Sud du territoire d'étude, la Zone E comprend les terres relativement plates des Laurentides, de l'Outaouais et d'AbitibiTémiscamingue. On y trouve des reliefs élevés tels que les monts Severson et MontTremblant $(968 \mathrm{~m})$. Cette zone est moins influencée par les vents océaniques ce qui explique la moyenne de $191 \mathrm{~mm}$ du maximum annuel de l'EEN. La zone F regroupe uniquement les Appalaches de la Gaspésie et du Bas-Saint Laurent. Au Nord, se trouvent les monts Albert (1154 m), Longue-Vue $(625 \mathrm{~m})$ et au Sud les piémonts. Du Nord au Sud, la partie est de la Zone F, présente un alignement de montagnes. La moyenne de $208 \mathrm{~mm}$ du maximum annuel moyen de l'EEN témoigne de la forte influence océanique sur les précipitations neigeuses. 
Les résultats de la validation de la segmentation spatiale au facteur d'échelle 30 de l'outil ESP sont présentés dans la Table 1. Les valeurs du maximum annuel moyen de l'EEN sont différentes d'une zone à l'autre excluant la Zone C. En effet, avec trois stations, la Zone C n'est pas incluse dans le test de comparaison. Sur le reste, les boxplots et les valeurs de $p(<0,05)$ confirment les différences entre les données des zones adjacentes. Par exemple, dans la zone A, l'EEN a une valeur maximale de 240 $\mathrm{mm}$ contre $398 \mathrm{~mm}$ dans la zone B. Les zones $\mathrm{E}$ et $\mathrm{F}$ ont des valeurs maximales respectives de 398 et $351 \mathrm{~mm}$, des minimas respectifs de 81 et $98 \mathrm{~mm}$. La valeur médiane de l'EEN est de $187 \mathrm{~mm}$ pour la Zone E et $200 \mathrm{~mm}$ pour la Zone F. Ces résultats de la segmentation spatiale se trouvent renforcés par comparaison aux études antérieures de classification des types de neige. Les limites de structures identifiées se rapprochent de celles de la moyenne de la densité de neige identifiées durant les périodes de février et mars (Brown 2014). De même, des similitudes apparaissent globalement entre les limites des structures régionales identifiées et les grandes zones climatiques de l'Est du Canada (MDDEFP 2001). Ceci confirme le rôle des grandes circulations atmosphériques dans la variabilité spatiale du couvert nival dans les hautes latitudes. Par ailleurs, les classifications de neige des maritimes et de la toundra de Sturm et al. (1995) correspondent approximativement aux zones géographiques aux structures homogènes $\mathrm{F}$ et $\mathrm{D}$ identifiées. La Zone $\mathrm{B}$ se rapproche de la classe de neige Taïga et plus à l'Ouest, la Zone A de la classe de neige de la Toundra (Sturm et al. 1995). En somme, les limites des structures identifiées se rapprochent des classes de neige et des découpages climatiques de l'Est du Canada.

Echelle locale - Analyse exploratoire des données La Fig. 9 montre les histogrammes, les diagrammes de dispersion ainsi que les coefficients de corrélations entre les variables statistiques du maximum annuel moyen de l'EEN 
et les variables physiographiques à l'échelle locale de la Zone D. Afin d'alléger le texte, ceux des autres zones étant similaires à ceux de la Zone D ne sont pas représentés. Les histogrammes des variables du maximum annuel moyen d'EEN ont en général des distributions moyennement symétriques. Les variables physiographiques locales telles que la pente, la distance aux lacs ont des distributions asymétriques à gauche. La radiation solaire, l'orientation et la hauteur des végétaux présentent des distributions bimodales. En effet, l'orientation présente deux zones distinctes des versants (ubac et adret) exposées différemment aux radiations solaires. La distribution des hauteurs des végétaux montre également une distribution bimodale du fait de la différence de taille entre les formations forestières et les zones de prairies. Les coefficients de corrélations sont en général faibles, mais très significatifs $(p<0,05)$. Le plus élevé s'observe entre la hauteur des végétaux et le maximum annuel moyen de l'EEN. Les formations forestières très développées sous le climat humide atlantique constituent d'excellents obstacles au transit des particules de neige par le vent humide de l'atlantique.

Méta-variables à l'échelle locale Les facteurs physiographiques locaux et les données de neige ont permis de calculer les méta-variables locales dans chacune des six zones géographiques selon l'équation (3). Les méta-variables $U_{1 L}$ ont des poids supérieurs aux poids des autres méta-variables dans toutes les zones. Dans la Zone A, la méta-variable $U_{1 L A}$ avec un poids $\left(\mathrm{P}_{\mathrm{U} 1 \mathrm{LA}}\right)$ de 0,41 présente des patrons spatiaux semblables à ceux des variables de la distance aux lacs, de la hauteur des végétaux et de la pente. La métavariable $U_{1 L \mathrm{~B}}$ de la Zone B avec un poids $\left(\mathrm{P}_{\mathrm{U} 1 \mathrm{LB}}\right)$ de 0,54 a des patrons spatiaux proches de ceux de la hauteur des végétaux et de la courbure des versants. Dans la Zone C, la méta-variable $U_{1 L C}$ se rapproche des patrons spatiaux de la radiation solaire, de la distance aux lacs et de la pente. Dans la Zone $\mathrm{D}$, la méta-variable $U_{1 L \mathrm{D}}$ présente des patrons spatiaux semblables à ceux de la hauteur des végétaux et de la pente. Dans la Zone $\mathrm{E}$, la méta-variable $U_{1 L \mathrm{E}}$ a des patrons spatiaux similaires à ceux des courbures des 
versants et de la pente tandis que la méta-variable $U_{1 L \mathrm{~F}}$ dans la Zone F se rapproche des patrons spatiaux des variables de la radiation solaire et de la distance aux lacs.

Segmentation spatiale à l'échelle locale A l'échelle locale, afin d'alléger le texte, les segmentations spatiales qui ne répondent pas au test de comparaison ne sont pas décrites. Dans la Zone A, quatre unités géographiques locales ont été identifiées au facteur d'échelle 300 par l'ESP (Fig. 10(a)). La segmentation spatiale est contrôlée par les pentes des bassins versants des principaux cours d'eau. On y trouve les forêts du Nord à densité moyenne à faible sous l'influence des vents humides des baies (Hudson et James). Au Nord, l'unité géographique (1) regroupe les espaces ouverts de tourbières. La seconde unité (2) présente des bassins versants aux pentes très faibles et des formes plus concaves. Au Sud, les unités géographiques (3 et 4) comportent également les formations basses de tourbières et d'arbustes par endroits.

Dans la Zone B, la segmentation spatiale est contrôlée principalement par la topographie complexe du Bouclier canadien, marquée à l'est et au centre par la dominance des plans d'eau (Fig. 10(b)). Au Nord, la Zone B englobe la ligne de contact entre les provinces géologiques du bouclier formant les monts Torngat et les lacs. En effet, les zones d'affaissement des failles correspondent aux unités hydrographiques distinctes telles que les lacs Mistissini, Manicouagan, Caniapiscau, Albanel, etc. A l'extrême Nord, les bassins versants des rivières sont plus convexes avec des fortes pentes sur les piémonts des monts Pingualuit, Qingaujaaluk (352 m) et Kanamikutau (440m). Au Sud, se distingue l'unité 10 incluant les vallées aux pentes élevées (59) des formations lithologiques intrusives et métamorphiques des monts La Tour $(579 \mathrm{~m})$, Chatigny $(579 \mathrm{~m})$ et Koaskiwonatinak $(663 \mathrm{~m})$. Au centre, les zones géographiques englobent les piémonts aux pentes abruptes des monts Otish $(1128 \mathrm{~m})$ et Tichegami 
(776 m). A l'Ouest, l'unité 8 située sur les basses terres se prolonge sur une grande partie de la Baie de James. Les formes des bassins versants sont globalement concaves. L'unité 8 s'étire du centre vers le Sud-Ouest regroupant plusieurs cours d'eau (Assinica, Nottaway etc.). A l'est, se suivent des unités géographiques $(1,3,4,5,6,7,9)$ regroupant les principaux grands monts du bouclier (Severson et Geren, 860m). Ces unités comportent les bassins versants des principaux cours d'eau des monts tels que Groulx et Babel (952 m), Veyrier (1104 m) et les piémonts des monts Otish et Yapeitso $(1128 \mathrm{~m})$. Ce sont des zones aux pentes abruptes et des versants globalement convexes. Dans l'ensemble, la Zone B est un domaine forestier allant des conifères de faible densité, aux forêts mixtes uniformes avec des peuplements de lichens, de fougères et d'arbustes au sol.

La segmentation spatiale de la Zone C détermine au facteur d'échelle 900 de l'ESP, quatre unités géographiques (Fig. 10(c)). Cette segmentation est contrôlée par la topographie élevée et complexe du Nord du territoire d'étude. En effet, le long de la mer de Labrador, les monts du Nord Torngat aux sommets élevés (Iberville, $1652 \mathrm{~m}$, Jacques-Rousseau, $1261 \mathrm{~m}$ ) coordonnent la dynamique de l'accumulation de la neige au sol. Les versants sont généralement abrupts et les pentes très fortes le long des plans d'eau. Le long des cours d'eau s'accumulent de fortes quantités de neige sous l'action du vent. La surface plane des eaux de surface accroit l'action du vent sur la mobilisation des particules de neige en fonction des obstacles rencontrés. Par ailleurs, la modification du gradient thermique de l'air par les lacs se remarque à des distances variables des berges sur le type de particules de neige. Par ailleurs, proche du pôle, comme il a été souligné plus haut, l'orientation des versants est un facteur important, car l'adret a un gradient thermique plus élevé que l'ubac et la neige s'y maintient moins longtemps. 
Dans la Zone D, la segmentation spatiale détermine au facteur d'échelle 200 de l'ESP, une vingtaine (23) de zones géographiques. Seules deux zones au Sud comportent le nombre suffisant de stations répondant au test de comparaison. La segmentation spatiale est contrôlée par la hauteur des végétaux, la distance aux lacs et la topographie complexe des bassins versants. De la Mer du Labrador jusqu'au Golfe du Saint-Laurent, tout au long de la façade atlantique, les cours d'eau descendant du Bouclier canadien vers les basses terres du Saint-Laurent ont des versants convexes (Fig. 10(d)). Ce sont des bassins versants aux pentes élevées dans les formations lithologiques intrusives et métamorphiques des monts (Kapathkatnahlu, Macatina, Brador, Grands Jardin). Des discontinuités spatiales hydrologiques entre les zones sont délimitées. En effet, on peut noter au Sud, le bassin versant de la rivière Saguenay entre l'unité 1 et l'unité 2 et au Nord-Est, le lac Melville dans les montagnes Mealy au Labrador. L'unité 1 et l'unité 2 sont des domaines de conifères à densité moyenne sous la forte influence des vents maritimes.

La segmentation spatiale de la Zone E est contrôlée par la topographie complexe et la hauteur des végétaux (Fig. 10(e)). Les unités géographiques déterminées englobent les piémonts aux versants abrupts des monts Chantigny $(579 \mathrm{~m})$ et des monts Grands Jardin. Les bassins versants aux pentes élevées et aux courbures convexo-concaves dominent le long des cours d'eau. C'est l'exemple de l'unité 8 englobant les MontsTremblant (998 m) et ses piémonts. Les pentes élevées et les versants abrupts sont des zones de transit des particules de neige, sous l'effet du vent et de la gravité, vers les secteurs concaves des versants. L'unité 9 regroupe les basses terres du fleuve SaintLaurent en contact avec le massif appalachien. Les unités 1, 4, 6 et 7 comportent les bassins versants des cours d'eau des monts Laurier et Sir Wilfried (783 m). Plus à l'Ouest, les unités géographiques locales regroupent les bassins versants aux pentes 
variables et aux courbures convexo-concaves des basses et hautes terres des Laurentides et de l'Outaouais. En général, la Zone E se caractérise par des forêts de conifères, de feuillus, des forêts mixtes intermédiaires et des terres agricoles ou en mosaïques. Cette mosaïque de formations végétales présente diverses oppositions à la mobilisation des particules de neige pour présenter des couvertures de neige plus ou moins épaisses au sol.

La segmentation spatiale de la Zone F est contrôlée par la topographie du relief appalachien et la hauteur des végétaux (Fig. 10(f)). En effet, au Nord, l'unité 1 regroupe les monts Chics-Chocs et Notre-Dame. Les versants apparaissent plus concaves ce qui correspond aux formes érodées du massif appalachien. Au Sud, l'unité 2 comprend une partie des terres basses du Saint-Laurent, les collines de Bécancour et les monts Suttononts Stoke du relief appalachien. La morphologie des bassins versants est relativement marquée par des pentes élevées avec des courbures convexo-concaves. Ce domaine est couvert de forêts mixtes et de terres agricoles. La présence de forêts mixtes et la prédominance des formes concaves des versants favorisent les divers types d'accumulation des particules de neige.

La validation de la segmentation spatiale de la Zone A (Table 2) montre que les distributions des données de neige sont différentes dans les unités 2 et 3 avec des médianes respectives de 216 et $116 \mathrm{~mm}$. Les données de neige dans l'unité 2 sont plus étalées et plus dissymétriques. La valeur significative de $p\left(10^{-3}\right)$ confirme la limite entre les deux structures spatiales des zones adjacentes. Dans la Zone B, les valeurs centrales des données de neige sont différentes d'une zone à l'autre (par exemple 328 mm dans l'unité 1 contre $277 \mathrm{~mm}$ dans l'unité 3) (Table 3). Les maxima annuels de l'EEN sont $398 \mathrm{~mm}$ dans l'unité 1 contre $294 \mathrm{~mm}$ dans l'unité 3. Les écarts interquartiles sont différents (par exemple 45 dans l'unité 1 contre 15 dans l'unité 3). 
D’une manière générale, les distributions sont dissymétriques d'une zone à l'autre. Cette tendance s'observe au niveau de toutes les distributions des données de neige des zones considérées. Dans la Zone $\mathrm{D}$, les distributions sont différentes d'une unité à l'autre avec des valeurs médianes différentes (281 mm pour l'unité 1 et $374 \mathrm{~mm}$ pour l'unité 2) (Table 4). Le maximum est de $367 \mathrm{~mm}$ dans l'unité 1 contre $515 \mathrm{~mm}$ dans l'unité 2. L'écart interquartile est de 130 dans l'unité 2 et de 20 dans l'unité 1 . La valeur significative de valeur $p\left(2 \times 10^{-3}\right)$ confirme la différence entre les données de neige des zones adjacentes. La Table 5 de la validation de la segmentation spatiale de la Zone E montre que les données de neige sont différentes d'une unité géographique à l'autre. Par exemple, la médiane est de $195 \mathrm{~mm}$ dans l'unité 3 et de $145 \mathrm{~mm}$ dans l'unité 6. La valeur maximale est de $207 \mathrm{~mm}$ dans l'unité 3 et de $160 \mathrm{~mm}$ dans l'unité 6. Dans la Table 6 de la validation de la segmentation de la Zone F, les maxima sont $351 \mathrm{~mm}$ et $266 \mathrm{~mm}$ dans l'unité 1 et l'unité 2. La valeur minimale est de $187 \mathrm{~mm}$ et de $98 \mathrm{~mm}$ respectivement dans les unités 1 et 2 . Ces valeurs confirment la différence entre les données du maximum annuel moyen dans les deux unités locales de la Zone F.

\section{CONCLUSION}

L'objectif principal de ce travail a été l'analyse multi-échelles de la variabilité spatiale de l'EEN à l'Est du Canada à l'aide de l'approche fonctionnelle. Cet objectif a été atteint sous l'hypothèse que le phénomène neige est stationnaire sur toute la période des observations. Dans un premier temps, les différentes structures de la variabilité spatiale du maximum annuel moyen de l'EEN ont été mises en évidence visuellement en fonction des positions géographiques (latitude et longitude) et quantitativement à l'aide de l'Indice d'Association Spatiale basé sur la similitude des valeurs des données des stations. Ces deux méthodes montrent que l'EEN n'est pas stationnaire à travers le territoire à l'étude, aussi bien au niveau de sa moyenne que de sa variance. Cependant, 
les limites des structures spatiales demeurent subjectives. Ce qui justifie le recours à la segmentation spatiale.

Par la suite, l'algorithme de segmentation spatiale a été appliqué pour délimiter explicitement les limites des structures de la variabilité spatiale de l'EEN. Cet algorithme a été appliqué sur les méta-variables physiographiques obtenues à l'aide de l'Analyse Canonique de Corrélation. Ces méta-variables physiographiques sont porteuses de l'information sur la variabilité spatiale de la neige à chaque échelle considérée puisqu'elles sont corrélées avec l'EEN. Ensuite, les résultats de la segmentation spatiale ont été validés, en comparant les données de neige des zones géographiques adjacentes, à l'aide du test statistique non paramétrique de KruskalWallis.

Ainsi, à l'échelle régionale, l'algorithme de segmentation spatiale multirésolutions a permis d'identifier six zones géographiques aux structures spatiales homogènes en termes d'EEN. Ces zones géographiques se rapprochent de la disposition des ensembles de relief (altitude) et des classes climatiques. A l'échelle locale, la segmentation spatiale a permis d'identifier plusieurs zones géographiques locales. A ce niveau d'observation, les structures locales identifiées de la variabilité spatiale de l'EEN démontrent le rôle dominant des facteurs physiographiques (pente, courbure, etc.) dans le maintien et la redistribution du couvert nival.

Par ailleurs, les résultats de cette étude posent les bases de deux analyses. La première base d'analyse est de porter un regard critique sur le réseau nivométrique actuel en termes de sa densité et de sa répartition spatiale. Ceci mène à l'identification des zones plus ou moins échantillonnées, les stations redondantes et à proposer une réorganisation optimale du réseau de stations nivométrique. Enfin la deuxième base d'analyse est l'amélioration des approches de spatialisation des paramètres physiques 
du couvert nival. Aussi, cette approche de segmentation spatiale peut servir dans un cadre prospectif si l'on dispose de données de l'EEN issues de simulation de modèle régional du climat (par exemple le Modèle Régional Canadien du Climat). Dans ce cas, on procèdera par une approche structurelle puisque l'on dispose des données de neige en tout point. Toutefois, les données simulées des modèles régionaux du climat sont à l'échelle régionale, ce qui ne permet pas de procéder à la segmentation spatiale à l'échelle locale. Les applications basées sur les simulations de données futures dépassent le cadre de ce projet.

Les travaux d'analyse de la variabilité spatiale du couvert nival suggèrent que les observations du maximum annuel de l'EEN ne sont pas stationnaires dans le temps (Brown 2010). Dans ce cas, il faudrait subdiviser les périodes d'observation en souspériodes pour lesquelles le maximum annuel moyen de l'EEN pourrait être considéré comme stationnaire. Par la suite, il faut reprendre la méthodologie de segmentation spatiale proposée dans cette étude. L'évolution des limites des structures serait alors analysée en fonction de la stationnarité de la neige. Par ailleurs, les résultats de cette étude se trouvent limités par la taille de l'échantillon. Cette dernière influence la qualité des résultats, car certaines limites de structures spatiales ne peuvent être confirmées en raison de l'absence ou de la taille très faible $(<4)$ de stations nivométriques dans certaines zones délimitées.

Remerciements Les auteurs remercient le Ministère du Développement Durable,

Environnement, de la Faune et des Parcs (MDDEFP), Environnement Canada, Rio Tinto et Hydro-Québec, pour les données d'équivalent en eau de la neige.

\section{REFERENCES}

Adler, P. B., Raff, D. A. et Lauenroth, W. K., 2001. The effect of grazing on the spatial heterogeneity of vegetation. Oecologia, 128, 465-479.

Alban, T., 2005. Application de l'approche orientée-objet à l'extraction de fragments forestiers à partir de scènes Spot. Université de Toulouse. Master Science de 
l'information Géoréférencée pour la Maîtrise de l'environnement et l'Aménagement des territoires

Anselin, L., 1995. Local indicators of spatial association (LISA). Geographical Analysis, 27(2), 93-115.

Baatz, M. et Schäpe, A., 2001. Multiresolution Segmentation: an optimization approach for high quality multi-scale image segmentation. In Angewandte geographische informationsverarbeitung, Edited by: Strobl, J., Blaschke, T. et Griesebner, G. 12-23. Heidelberg: Wichmann-Verlag.

Baccini, A. et Besse, P., 2004. Data mining. Exploration Statistique. Toulouse III: Université Paul Sabatier. 142 p.

Beltrando, G. et Chémary, L., 1995. Dictionnaire du climat Paris. 344 p.

Ben Arfa, N., Rodriguez, C. et Daniel, K., 2008. Dynamiques spatiales de la production agricole en France 2èmes journées de recherches en sciences sociales INRA SFER CIRAD. France, 25.

Braateen, R. O. et Brown, R. D., 1998. Spatial and temporal variability of Canadian monthly snow depths , 1946-1995. Atmosphère-Océan, 36(1), 37-54.

Brasnett, B., 1999. A global analysis of snow depth for numerical weather prediction. Journal of Applied Meteorology, 38(6), 726-740.

Brown, R., 2014. Mean snow density from snow courses in Feb, March, April (19661985). unpublished.

Brown, R., Derksen, C. et Wang, L., 2007. Assessment of spring snow cover duration variability over northern Canada from satellite datasets. Remote Sensing of Environment, 111(2-3), 67-381.

Brown, R. D., 2000. Northern Hemisphere Snow Cover Variability and Change, 19151997. Journal of Climate, 13(13), 2339-2355.

Brown, R. D., 2010. Analysis of snow cover variability and change in Québec, 19482005. Hydrological Processes.

Brown, R. D. et Goodison, B. E., 1996. Interannual variability in reconstructed Canadian snow cover, 1915-1992. Journal of Climate, 9(6), 1299-1318.

Byrd, G., 2000. Lake-Effet Snow [online]. Ontario. Available from: http://www.comet.ucar.edu/class/smfaculty/byrd/index.htm [Accessed Janvier 2012].

Caillault, S., Ballouche, A. et Delahaye, D., 2009. Organisation spatio-temporelle des feux de brousse. Approche comparative au Burkina Faso Neuvièmes Rencontres de Théorie Quantitative Besançon-France.

Carroll, S. S. et Cressie, N., 1997. Spatial modeling of snow water equivalent using covariances estimated from spatial and geomorphic attributes. Journal of Hydrology, 190(1-2), 42-59.

CCT, 1999. Centre Canadien de Télédétection. Atlas Canada. Ressources naturelles Canada. Canada Couverture des terres. Ottawa: MCR 103F, Série de cartes de référence de l'Atlas national du Canada.

Chokmani, K., Bernier, M. et Gauthier, Y., 2006. Suivi spatio-temporel du couvert nival du Québec à l'aide des données NOAA-AVHRR. Revue des sciences de l'eau / Journal of Water Science, 19(3), 163-179.

Chokmani, K., Bernier, M. et Slivitzky, M., 2005. Validation of a method for snow cover extent monitoring over Quebec (Canada) using NOAA-AVHRR data. EARSE Le Proceedings, 4 (1), 106-118.

Corbel, J., 1958. La neige dans les régions hautement polaires (Canada, Groenland) audelà du $80^{\circ}$ latitude Nord. Revue de géographie alpine, 343-366. 
Croux, A. et Dehon, B., 2003. Analyse canonique basée sur des estimateurs robustes de la matrice de covariance. Université Libre de Bruxelles.

Daly, C., Neilson, R. P. et Phillips, D. L., 1994. A statistical-topographic model for mapping climatological precipitation over mountainous terrain Journal Applied Meteorology, 3, 140-158.

De Sève, D., et al., 2001. Estimation de l'équivalent en eau de la neige au sol dans un milieu de taiga à l'aide des données SSM/I. Télédétection, 2(1), 13-28.

Dickison, R. B. B. et Daugharty, D. A., 1977. Effects of forest cover and topography on snow cover in the Nashwaak experimental watershed project. Proc. 2nd Conference on Hydrometeorology. Toronto 245-250.

Dodge, Y., 2007. Statistique. Dictionnaire encyclopédique. Springer Paris. p.

Drăguţ, L. et Eisank, C., 2011. Object representations at multiple scales from digital elevation models. Geomorphology, 129, 183-189.

Drăguţ, L., et al., 2010. ESP: a tool to estimate scale parameter for multiresolution image segmentation of remotely sensed data. International Journal of Geographical Information Science, 24(6), 859-871.

Dyer, J., 2008. Snow depth and streamflow relationships in large North American watersheds. Journal of Geophysical Research, 113(18).

Eichler, T. et Higgins, W., 2006 Climatology and ENSO-related variability of North America extratropical cyclone activity Journal of Climate, 19, 2076-2093.

Erxleben, J., Elder, K. et Davis, R., 2002. Comparison of spatial interpolation methods for estimating snow distribution in the Colorado Rocky Mountains. Hydrological Processes, 16(18), 3627-3649.

Evora, N. D., Tapsoba, D. et De Sève, D., 2008. Combining artificial neural network models, geostatistics, and passive microwave data for snow water equivalent retrieval and mapping. IEEE Transactions on Geoscience and Remote Sensing, 46(7), 1925-1939.

Fillon, L. et Payette, S., 1976. La dynamique de l'enneigement en région hémi-arctique, Poste-de-la-Baleine, Nouveau-Québec. Cahiers de Géographie de Québec, 20(50), 275-302.

Fortin, J. P., Morin, G. et Dupont, L., 1982. Variation saisonnière de l'homogénéité spatiale et de la structure régionales de la pluie au Québec Atmosphère-Océan, $20(3), 227-241$.

Garrigues, S., 2004. Hétérogénéité spatiale des surfaces terrestres en télédétecttion: caractérisation et influence sur l'estimation des variables biophysiques. (3ième cycle). Université de Rennes

Goïta, K., Walker, A. E. et Goodison, B. E., 2003. Algorithm development and use for the estimation of snow water equivalent in the boreal forest using passive microwave data. International Journal of Remote Sensing, 24(3), 1097-1102

Goovaerts, P., 2000. Geostatistical approaches for incorporating elevation into the spatial interpolation of rainfall. Journal of Hydrology, 228 (1-2), 113-129.

Gray, D. M. et Male , D. H., 1981. Handbook of Snow. 776 p.

Guay, J. F., et al., 1998. Les oscillations climatiques à moyennes fréquences: causes possibles, mécanismes et effets connus au Canada et au Québec. Québec: INRS, R000538. Rapport annuel 1998-1999.

Haché, M., et al., 2002. Estimation régionale par la méthode de l'analyse canonique des corrélations : comparaison des types de variables hydrologiques. Can, J. Civ Eng., 29, 899-910.

Hantel, M., Ehrendorfer, M. et Haslinger, A., 2000. Climate sensitivity of snow cover duration in Austria. International Journal of Climatology, 20, 615-640. 
IFNC., 2004. Inventaire forestier national du Canada. Schéma de classification de la couverture terrestre [online]. Available from: https://nfi.nfis.org/photo_plot.php?lang = fr [Accessed Février 2013].

Jones, H. G., et al., 2001. Snow Ecology. Cambridge University Press. 450 p.

Kronholm, K. et Birkeland, K., W., 2007. Reliability of sampling designs for spatial snow surveys. Computer and Geosciences, 33, 1097-1110.

Laffitte, P. et Marc., A., 2010. Méthodes d'analyse des autocorrélations spatiales Département d'Enseignement et de Recherche en Informatique et Technologies Urbaines, EIVP 54.

Leduc, R. et Gervais, R., 1985. Connaître la météorologie. Montréal p.

Levin, S. A., 1992. The problem of pattern and scale in ecology Ecology, 73(19431967).

Lhotellier, R., 2005. Spatialisation des températures en zone de montagne alpine. (3 ième cycle). Université Joseph Fourier-Grenoble 1.

Li. et Reynolds, J. F., 1995. On definition and quantification of heterogeneity. Oikos, 73(2), 280-284.

Marsh, P., 1999. Snow cover formation and melt: recent advances and future prospects. Hydrological Processes, 13(14-15 ), 2117-2134.

Marty, C., 2008. Regime shift of snow days in Switzerland. Geophysical Research Letters, 35(12), 5.

McKay, G. A. et Gray, D. M., 1981. Distribution of snow cover in Handbook of Snow. Eds., Pergamon Press 153-190 p.

MDDELCC, 2001. Contribution du Service de la cartographie écologique: Une classification climatique du Québec à partir de modèles de distribution spatiale de données climatiques mensuelles : vers une définition des bioclimats du Québec [online]. Québec: Ministère du Développement Durable, de l'Environnement de la Lutte contre les Changements Climatiques. Available from: $\quad$ http://www.mddep.gouv.qc.ca/changements/classification/modelclima.pdf [Accessed Février 2012].

MDDELCC, 2002. Ministère du Développement Durable, de l'Environnement de la Lutte contre les Changements Climatiques: Aires protégées au Québec et les provinces naturelles [online]. Available from: http://mddefp.gouv.qc.ca/biodiversite/aires_protegees/provinces/partie3.htm\#reli ef [Accessed Février 2012].

MDDELCC, 2008. Manuel d'instructions à l'usage des observateurs en nivométrie, Québec. Québec: Ministère du Développement Durable, de l'Environnement de la Lutte contre les Changements Climatiques, 978-2-550-52028-3.

Morency, C., 2006. Étude de méthodes d'analyses spatiales et illustration à l'aide de l'aide de micro données urbaines de la grande région de Montréal Les Cahiers Scientifiques du Transport, 49, 77-102.

Mote, P. W., et al., 2005. Declining mountain snowpack in western North America. Bulletin of the American Meteorological Society, 86, 39-49.

MRN., 2013. Ministère des Ressources Naturelles: Zones de végétation et domaines bioclimatiques $d u$ Québec [online]. Available from: http://www.mrn.gouv.qc.ca/forets/inventaire/inventaire-zones-carte.jsp [Accessed Décembre 2013].

MSC., 2004. Aerological Observer's Course. Module 2.5-Snow Survey. Environnement Canada, Service Météorologie du Canada, 43.

Nagao, M. et Matsuyama, T., 1979. Edge preserving smoothing. Computer graphics and image processing, 9, 394-407. 
Oetlli, P. et Camberlin, P., 2005. Influence of topography on monthly rainfall distribution over East Africa. Climate Research, 28, 199-212

Palacios, D. et Sánchez-Colomer, M. G., 1997. The distribution of high mountain vegetation in relation to snow cover: Peñalara Spain CATENA, 30(1), 1-40.

Perrier, R., 1968. Les pluies maximales probables au Québec Cahiers de géographie du Québec, 12(27), 421-428.

Plamondon, A. P., Prévost, M. et Naud, R. C., 1984. Accumulation et fonte de la neige en milieux boisé et déboisé. Géographie physique et Quaternaire, 38(1), 27-35.

Poinsot, D., 2004. Statistiques pour statophobes [online]. Available from: http://perso.univ-rennes1.fr/denis.poinsot [Accessed Octobre 2012].

Rasmussen, P., et al., 1999. Impact des oscillations climatiques a basse fréquence sur les apports des rivières québécoises: Etude statistique exploratoire. . Research Report R-541, Institut national de recherche scientifique, INRS-Eau, Sainte-Foy, Québec, July, 1999, 60.

RNC, 2000. Ressources naturelles Canada. Données numériques d'élévation du Canada [online]. Sherbrooke (Québec), Canada. Available from: http://www.geobase.ca/geobase/en/find.do?produit $=$ cded $\quad$ [Accessed Juin 2010].

Sobolowski, S. et Frei, A., 2007. Lagged relationships between North American snow mass and atmospheric teleconnection indices. International Journal of Climatology 27, 221-231.

Sturm, M., Holmgren, J. et Liston, G. E., 1995. A Seasonal Snow Cover Classification System for Local to Global Applications. Journal of Climate, 8(5), 1261-1283.

Swanson, R. H., 1970. Local snow distribution is not function of local topography under continous tree cover Journal of Hydrology, 9(2), 292-298.

Turcotte, R., et al., 2006. Operational analysis of the spatial distribution and the temporal evolution of the snowpack water equivalent in southern Québec, Canada. Nordic Hydrology, 38 (3), 211-234.

Vinatier, S., 2008. Compléments de Mathématique. In: Limoges, F. d. S. e. T. d. ed. Licence de Biologie, 3ième semestre. Cours de Mathématiques, 3.

Watson, F. G. R. et Anderson, T. N., 2006. Optimal sampling schemes for estimating mean snow water equivalents in stratified heterogeneous landscapes Journal of Hydrology, 328(3-4), 432-452. 
Figure 1 Localisation géographique du territoire d'étude.

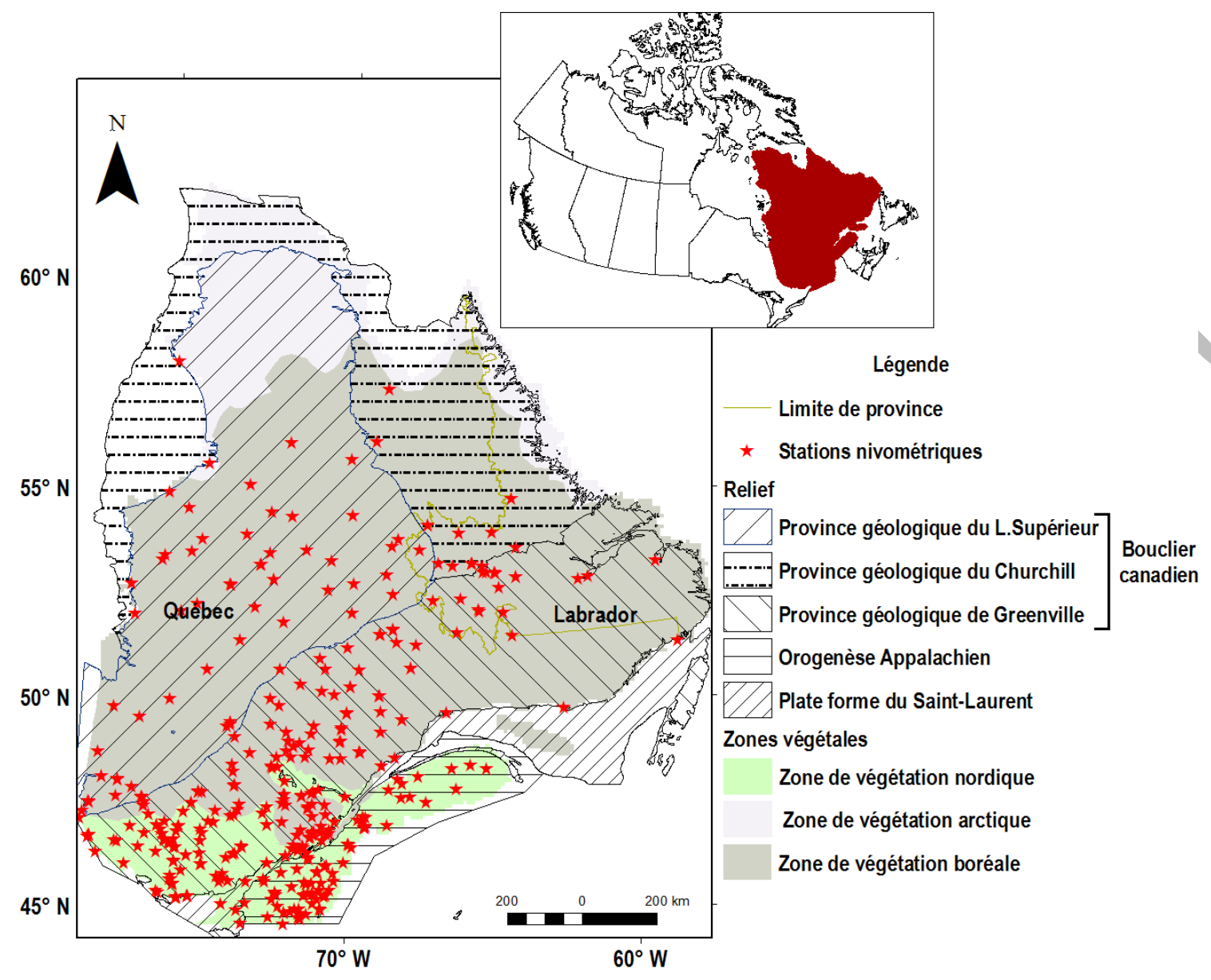


Figure 2 Méthodologie de la segmentation spatiale.

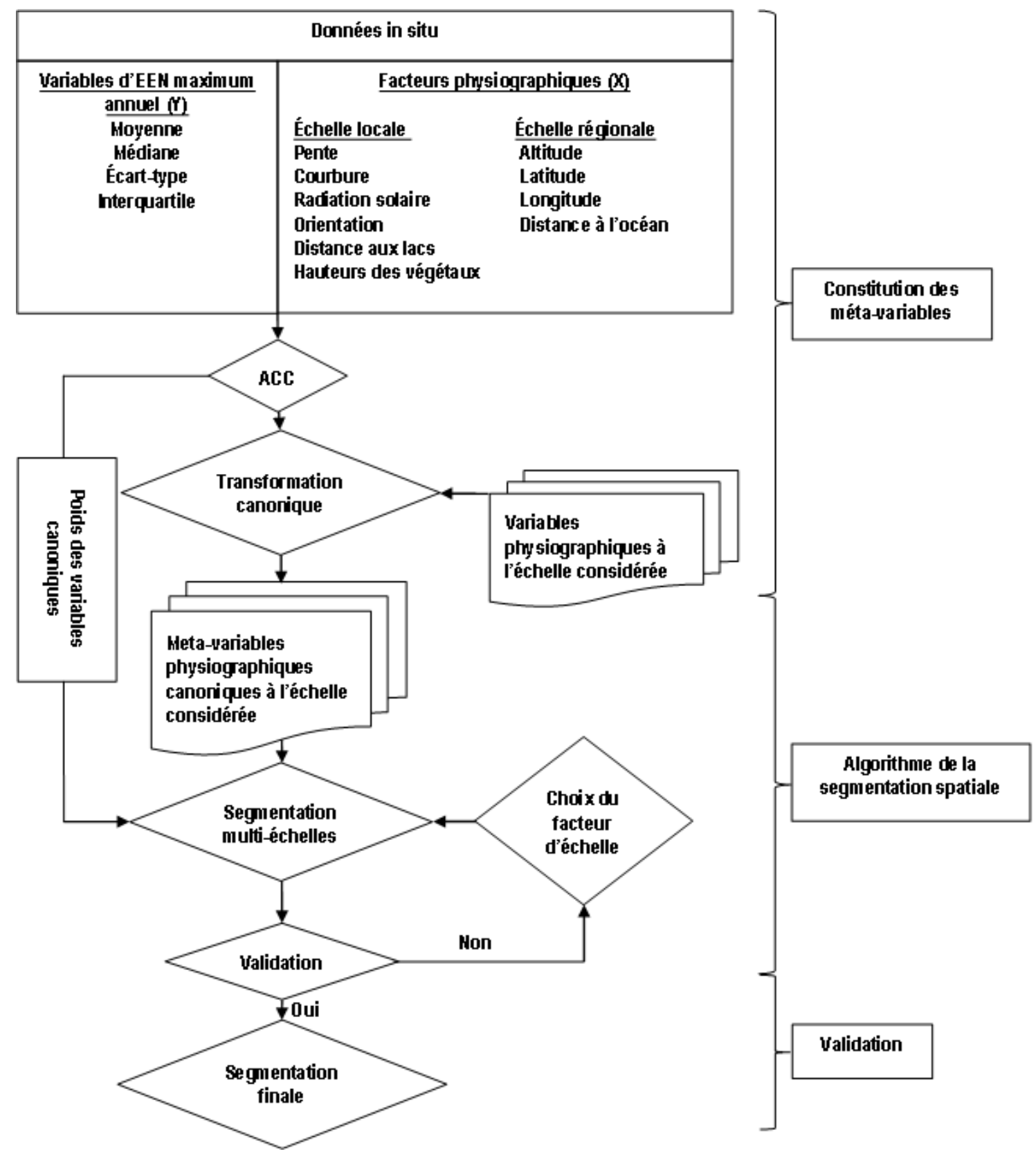


Figure 3 Hétérogénéité des structures de la variabilité spatiale de l'EEN selon la longitude (a) et la latitude (b).

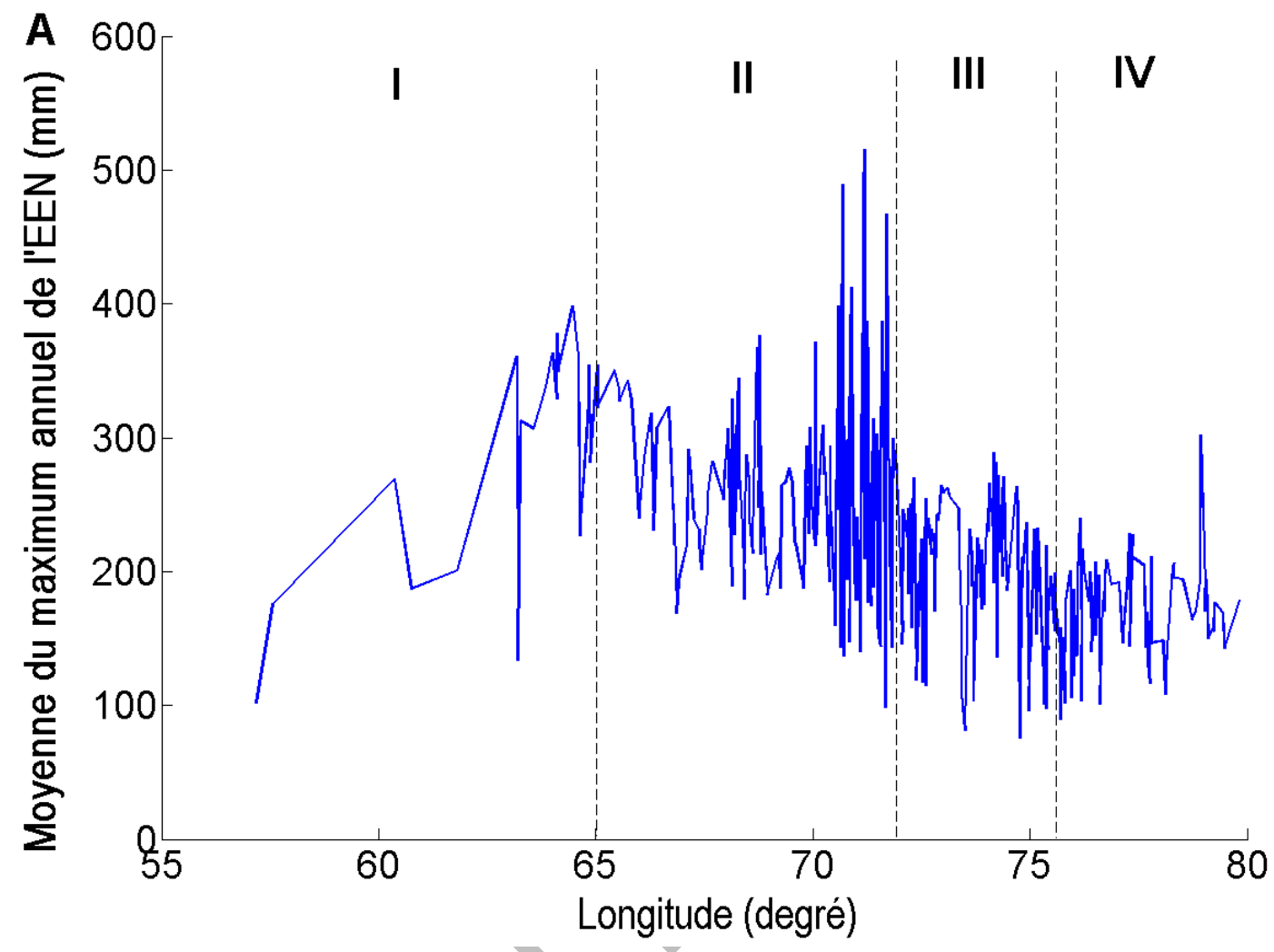




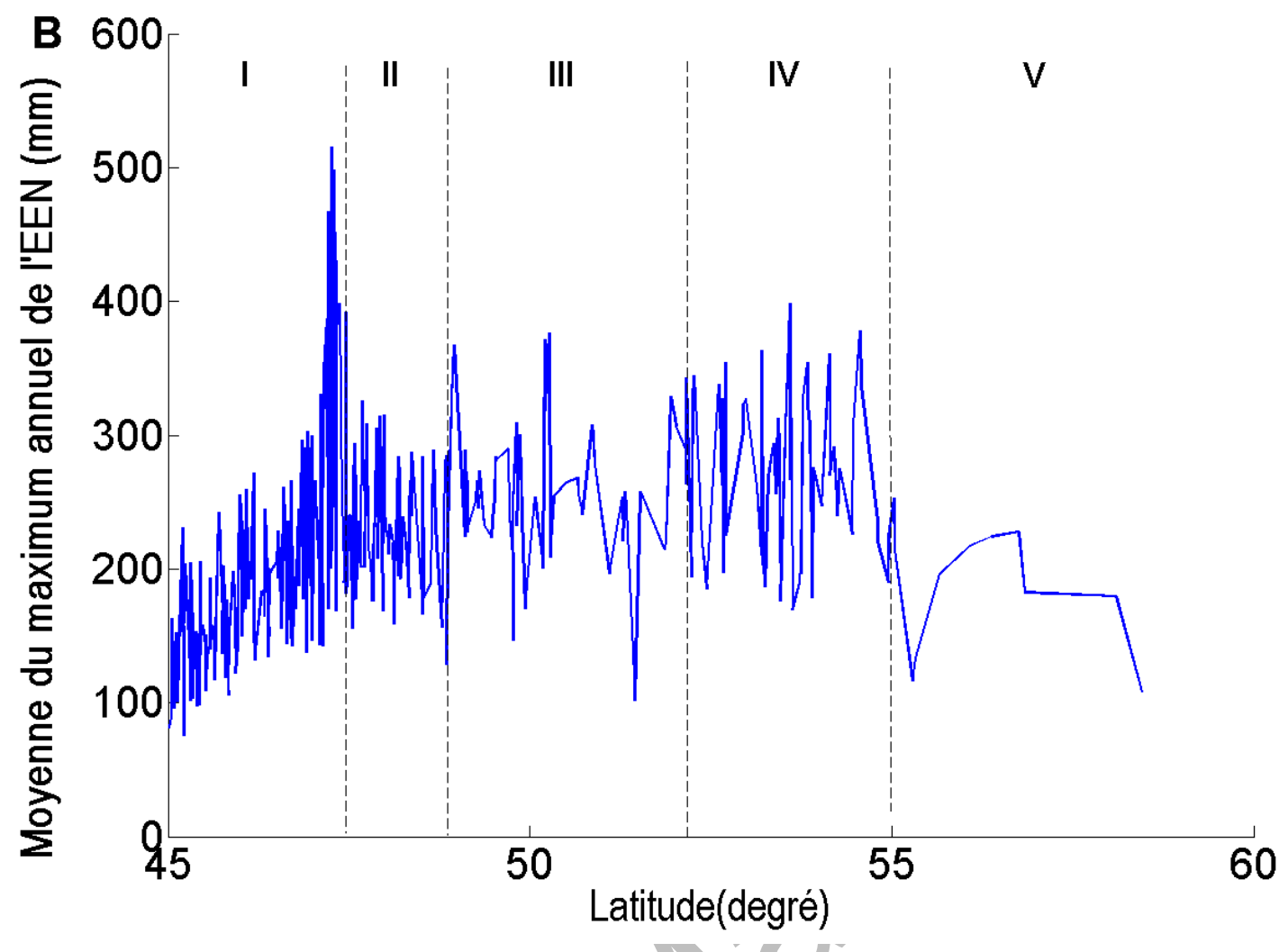


Figure 4 Degré d'association spatiale selon l'indicateur local d'association spatiale d'Anselin (1994).

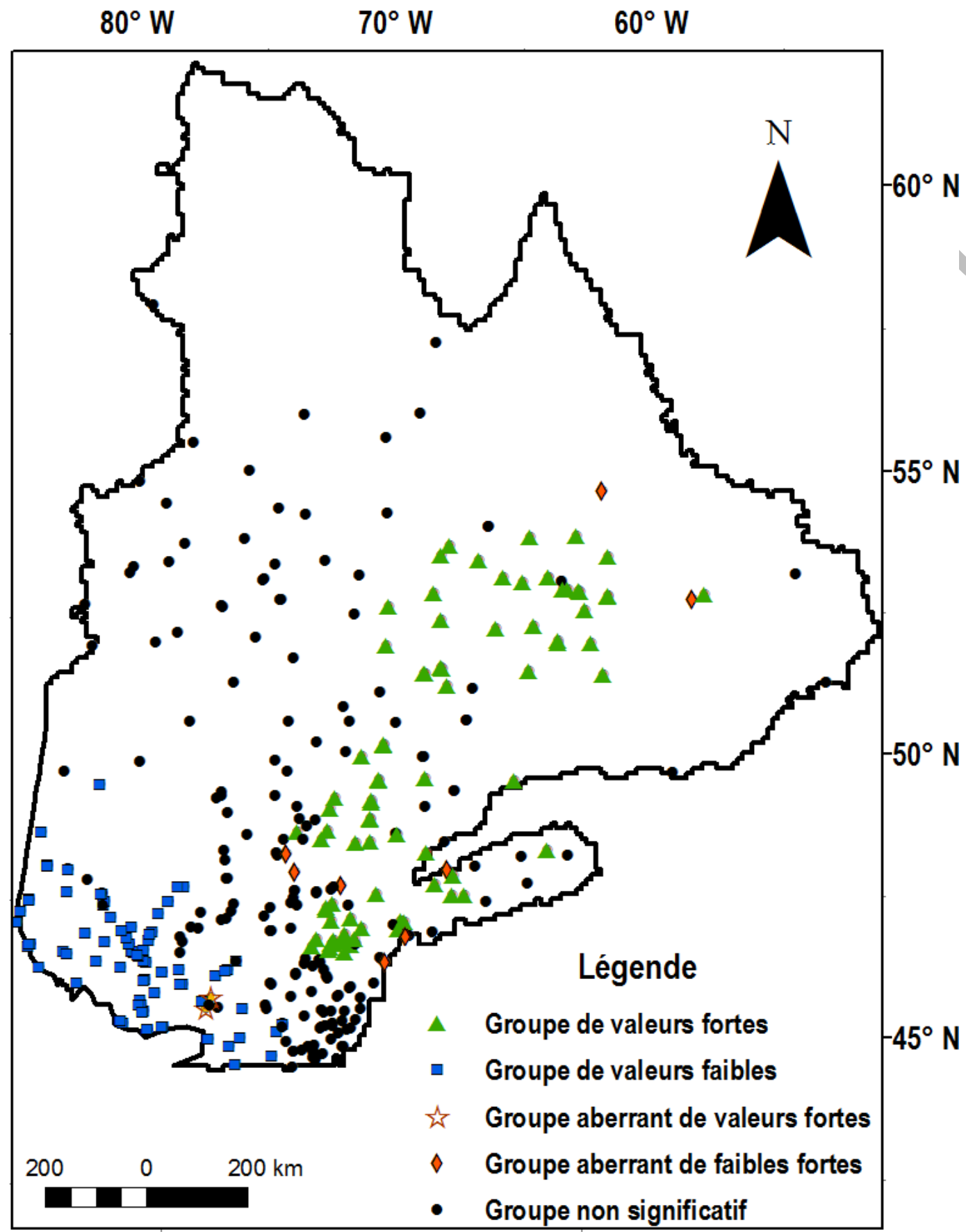


Figure 5 Corrélation entre les facteurs physiographiques régionaux et les variables de l'EEN.

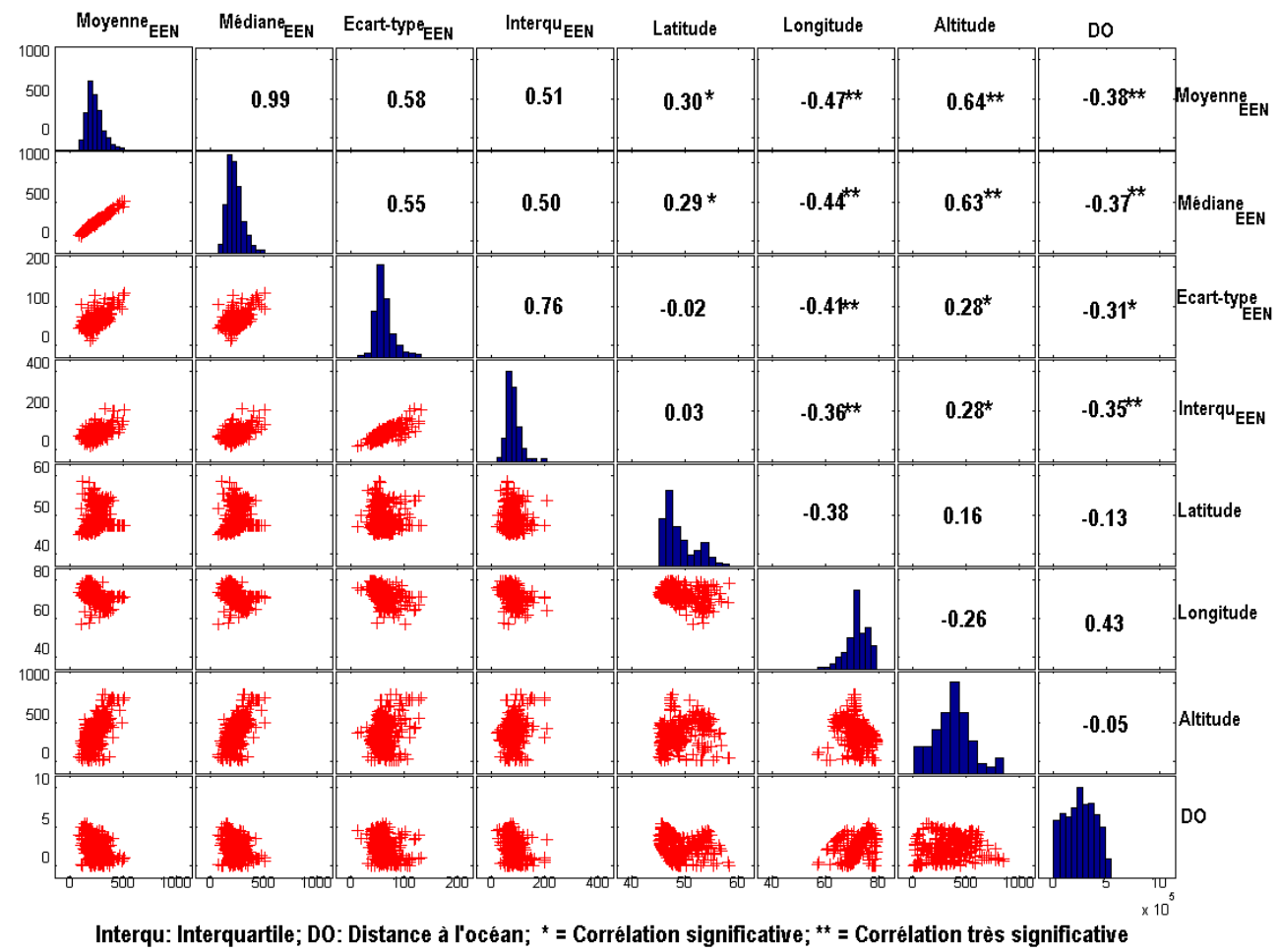


Figure 6 Méta-variables physiographiques obtenus par la méthode d'analyse canonique de corrélation à l'échelle régionale (a:U1; b: U2, c: U3; d: U4).

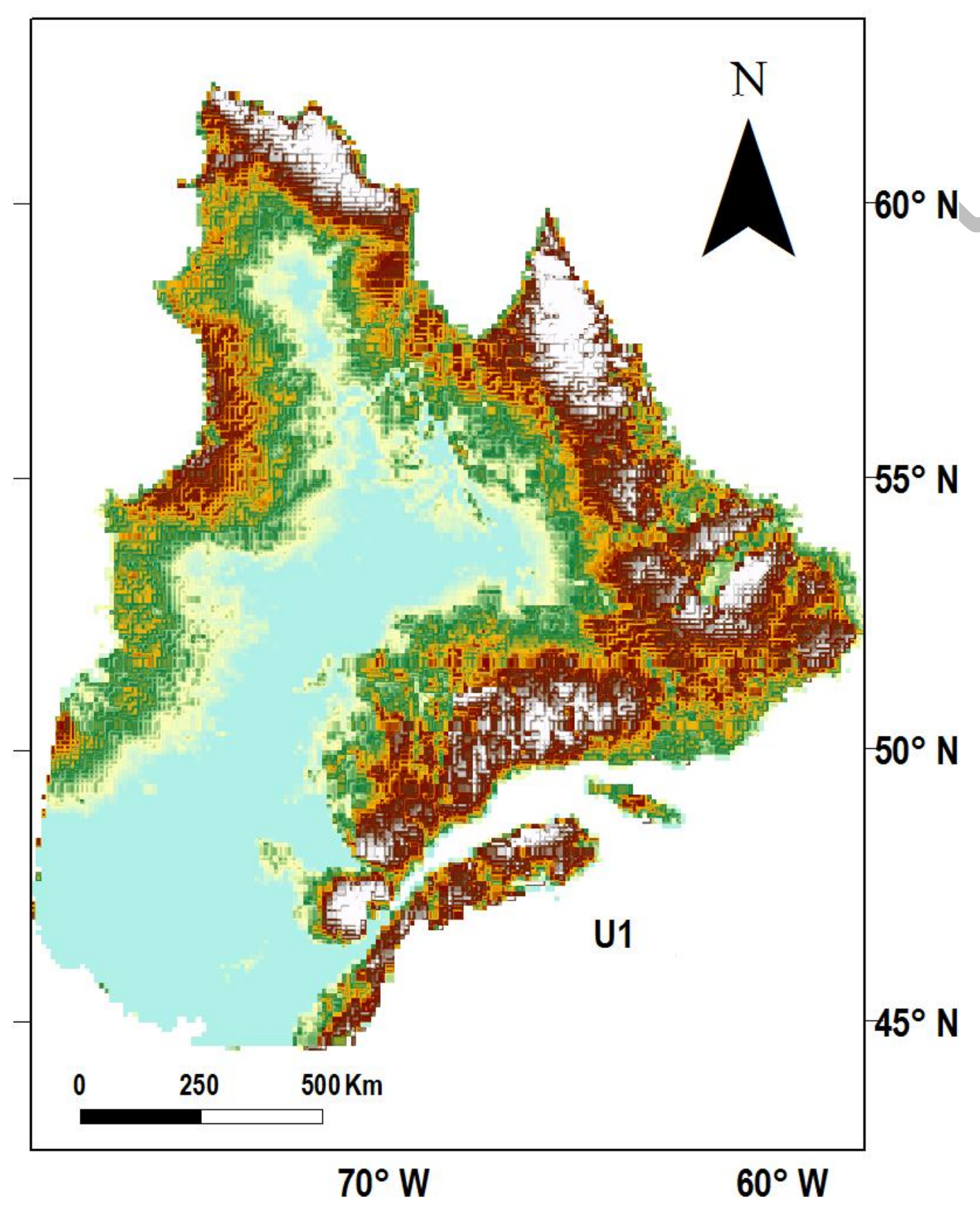




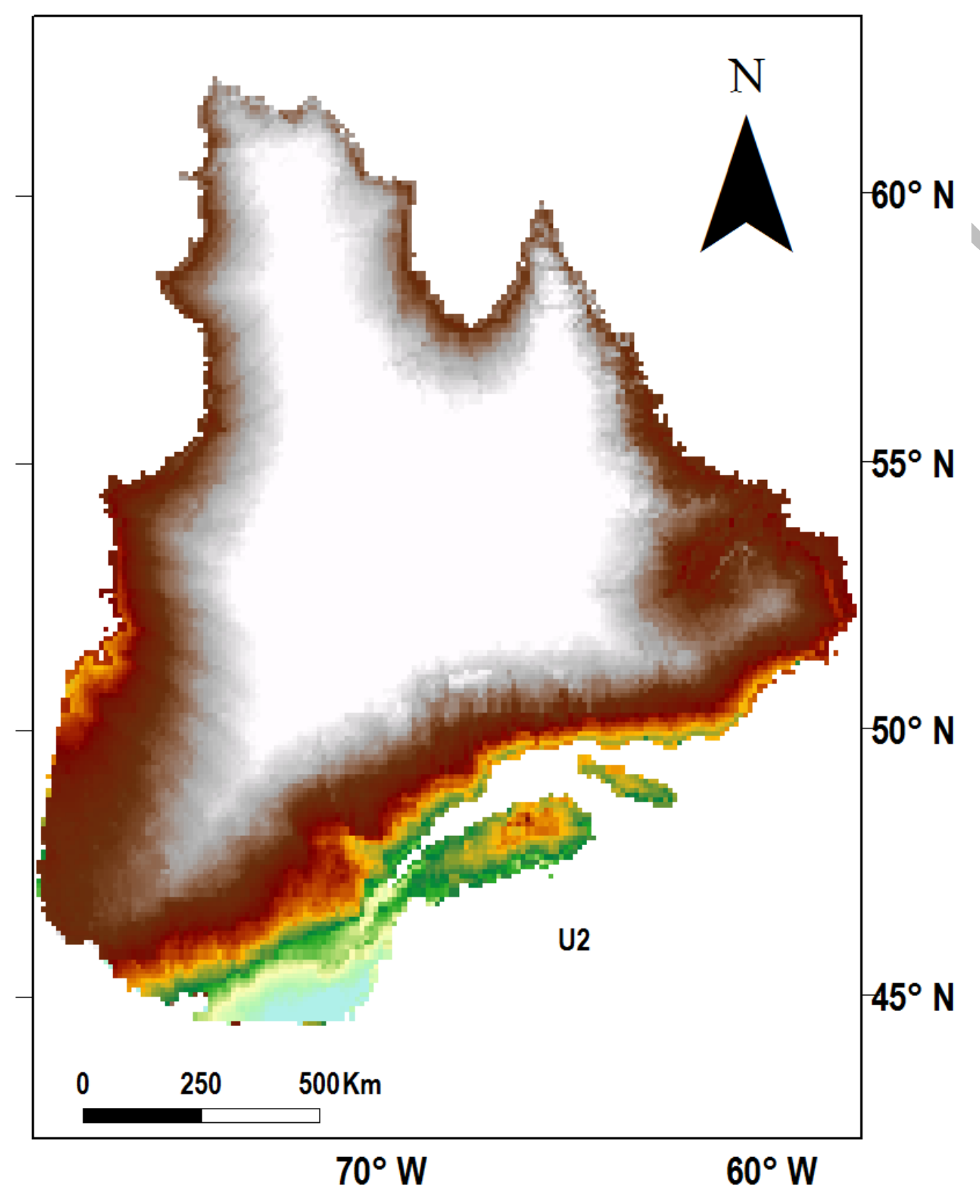




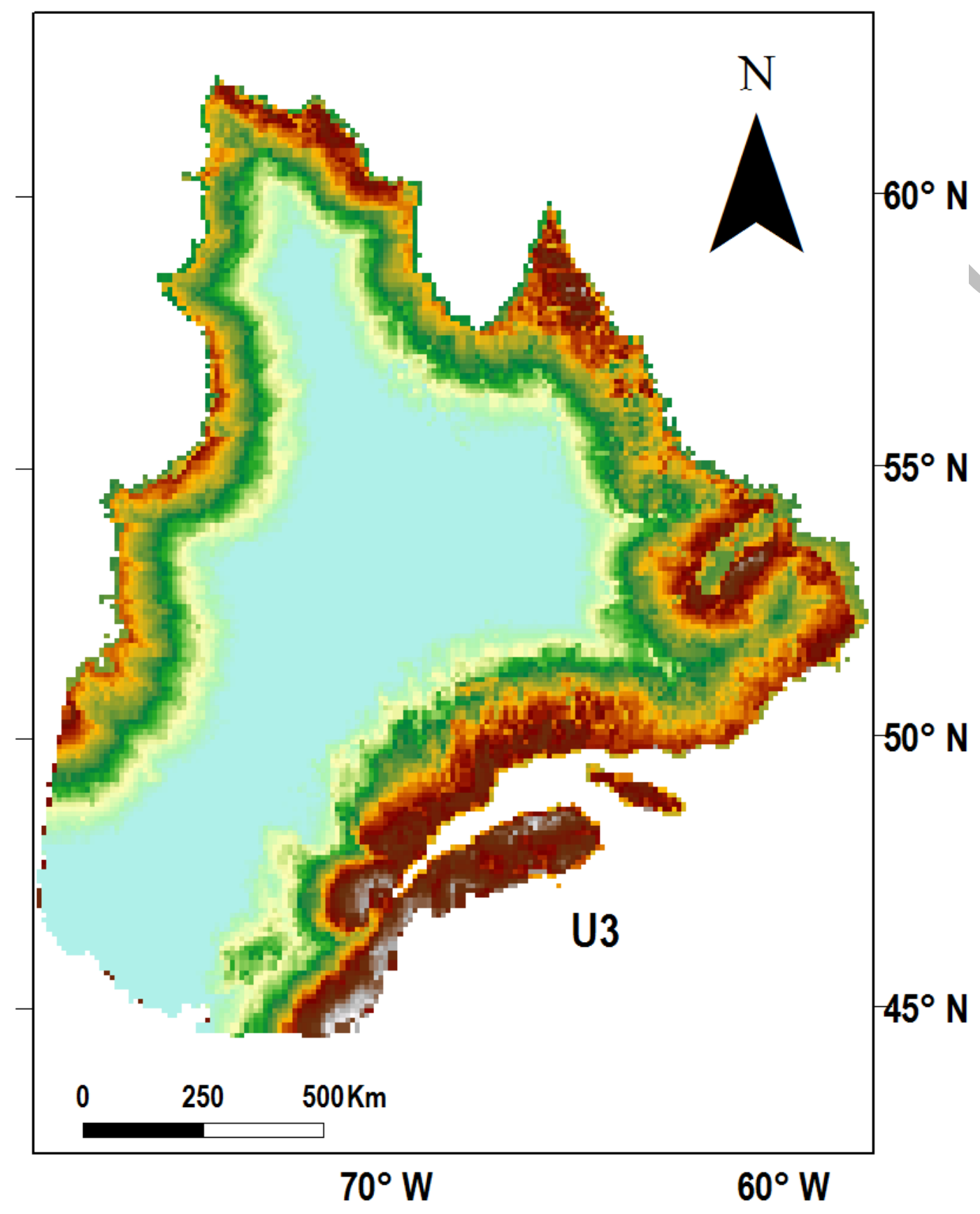




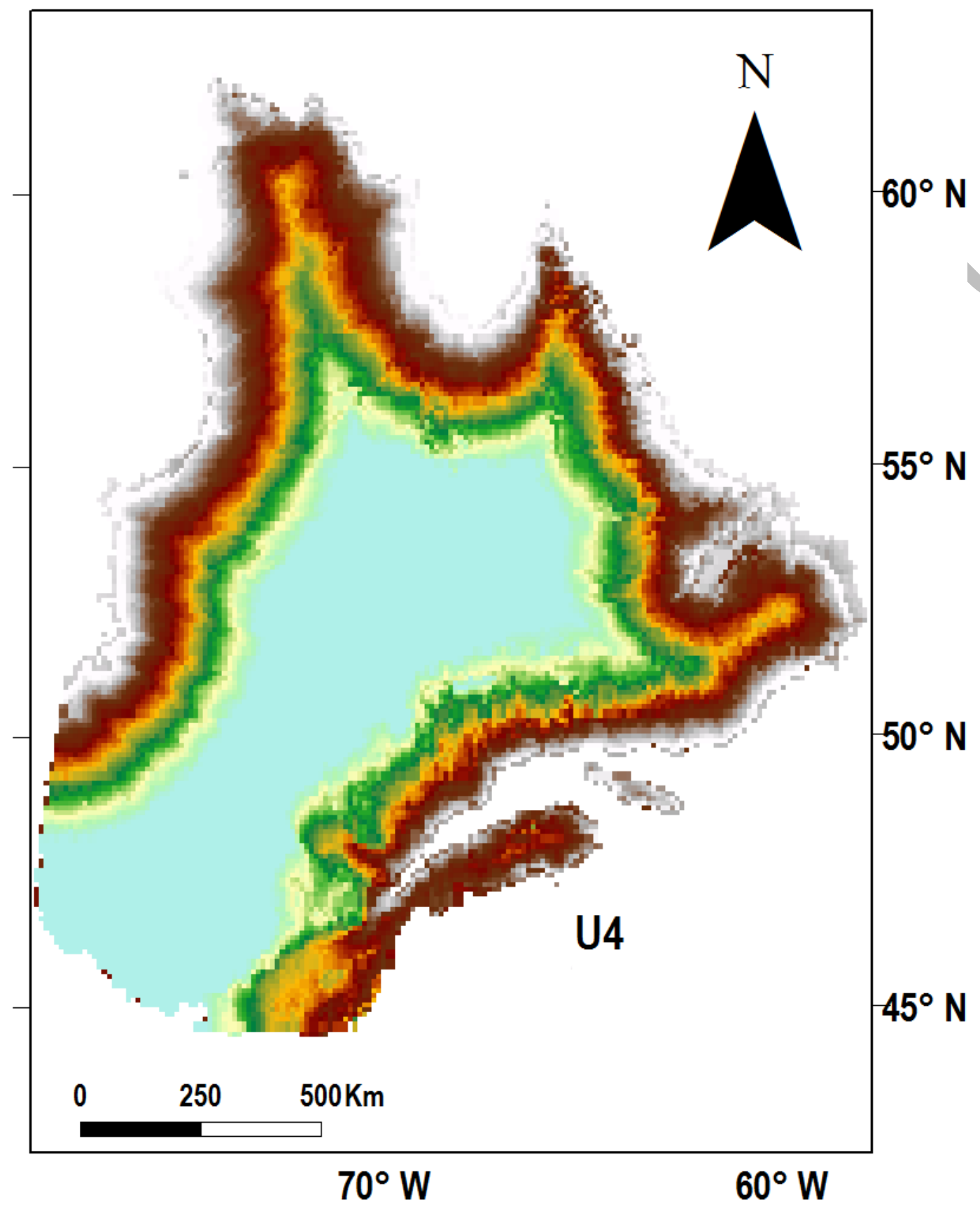


Figure 7 Application de l'outil d'ESP (Drăguţ et al. 2010) pour la détermination des différents facteurs d'échelle de la segmentation spatiale à l'échelle régionale.

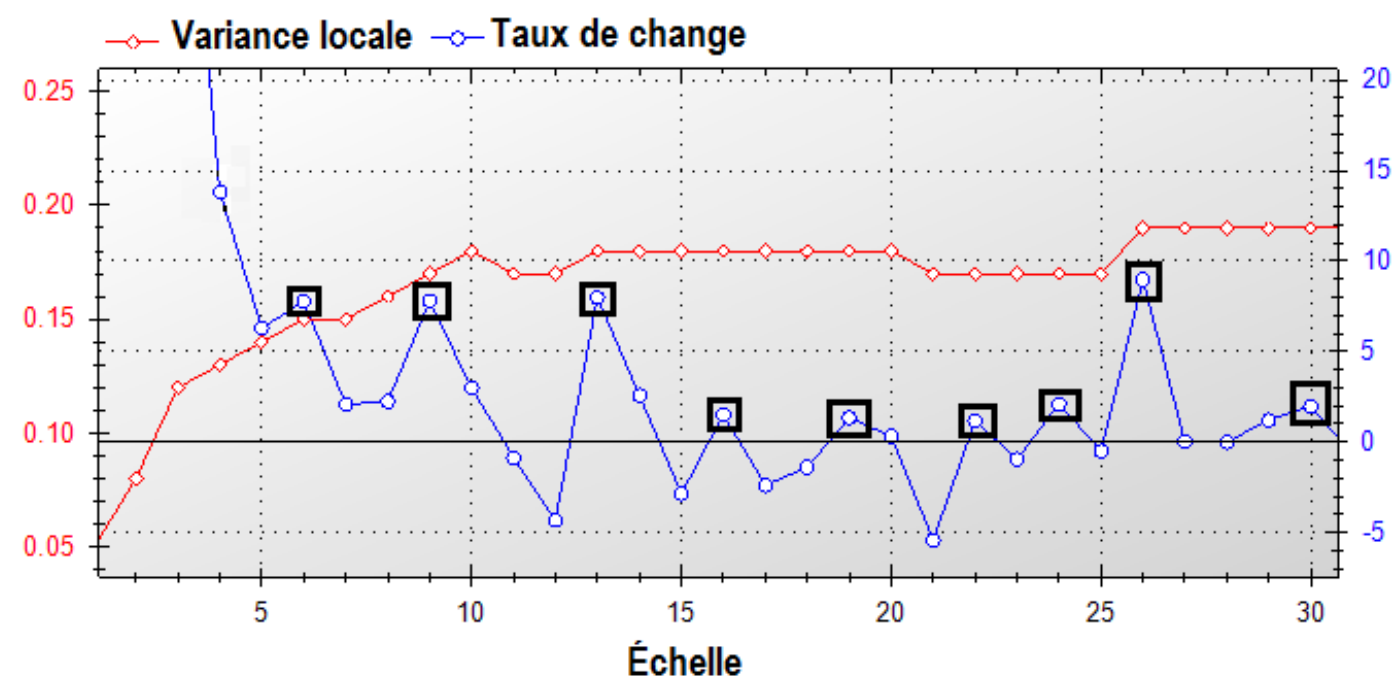


Figure 8 Zones géographiques aux structures spatiales homogènes à l'échelle régionale

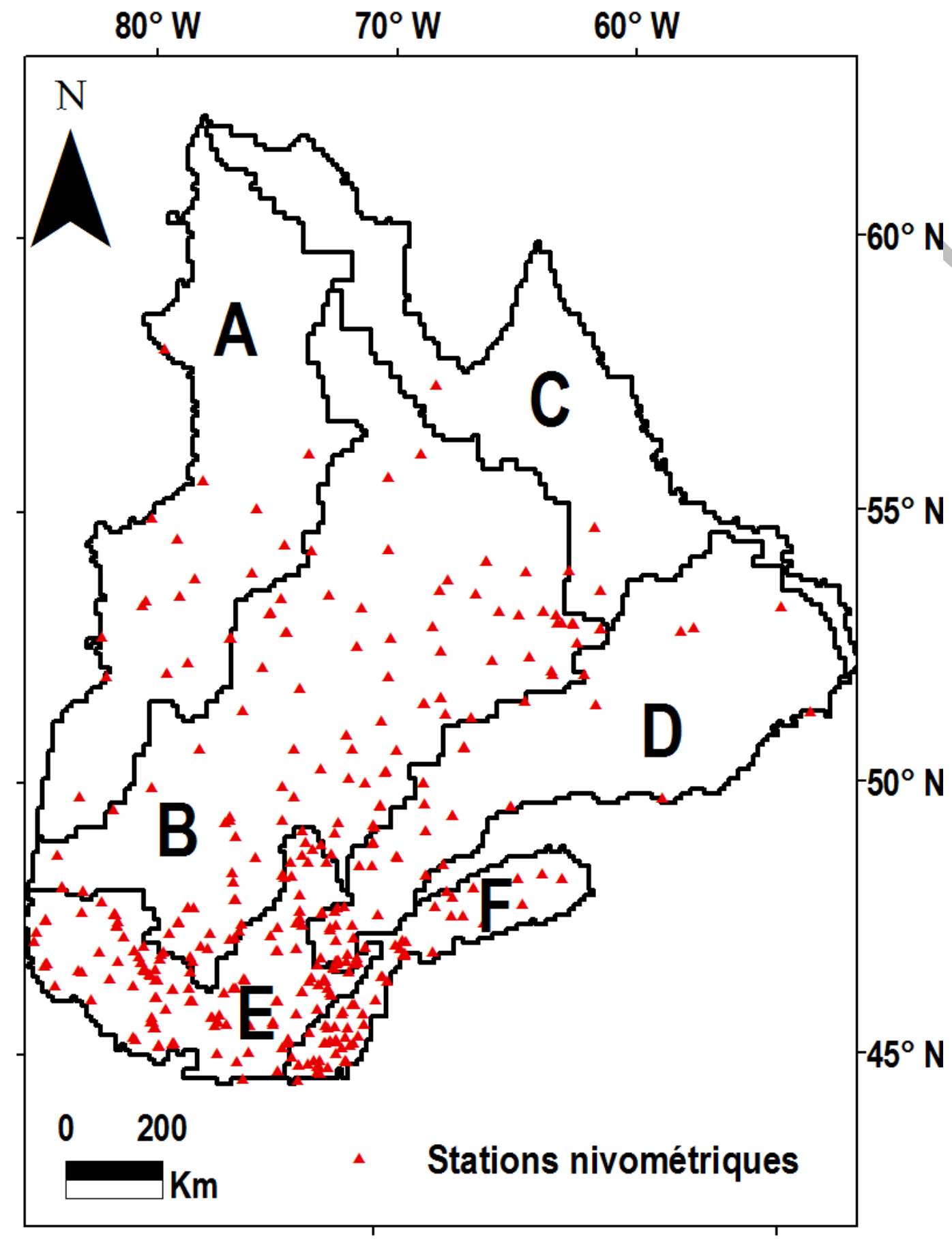


Figure 9 Corrélation entre les facteurs physiographiques locaux et les variables de l'EEN de la zone D.

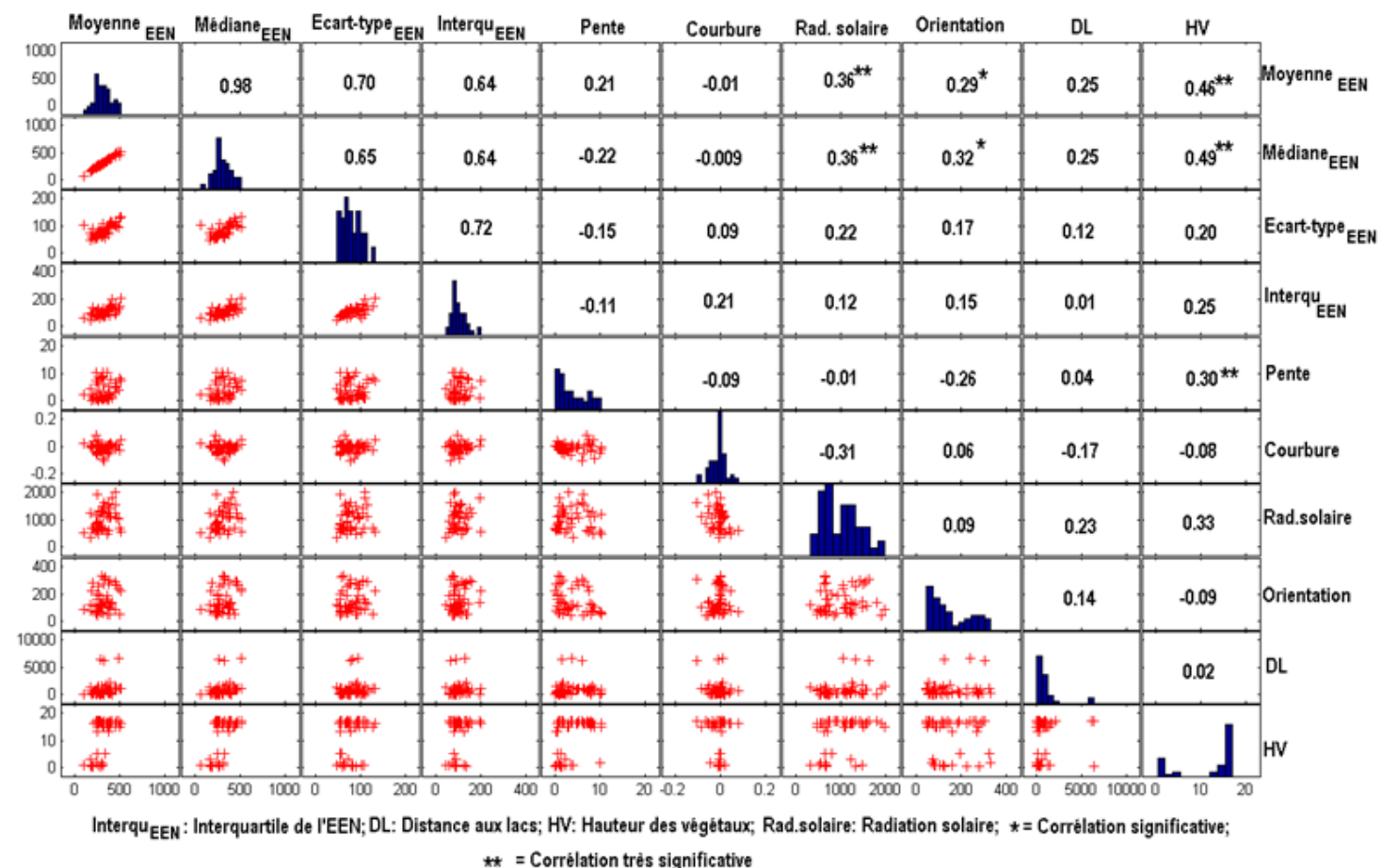


Figure 10 Zones géographiques aux structures spatiales homogènes à l'échelle locale dans la zone A(a); la zone B (b); la zone C (c); la zone D (d); la zone E (e) et la zone F (f).

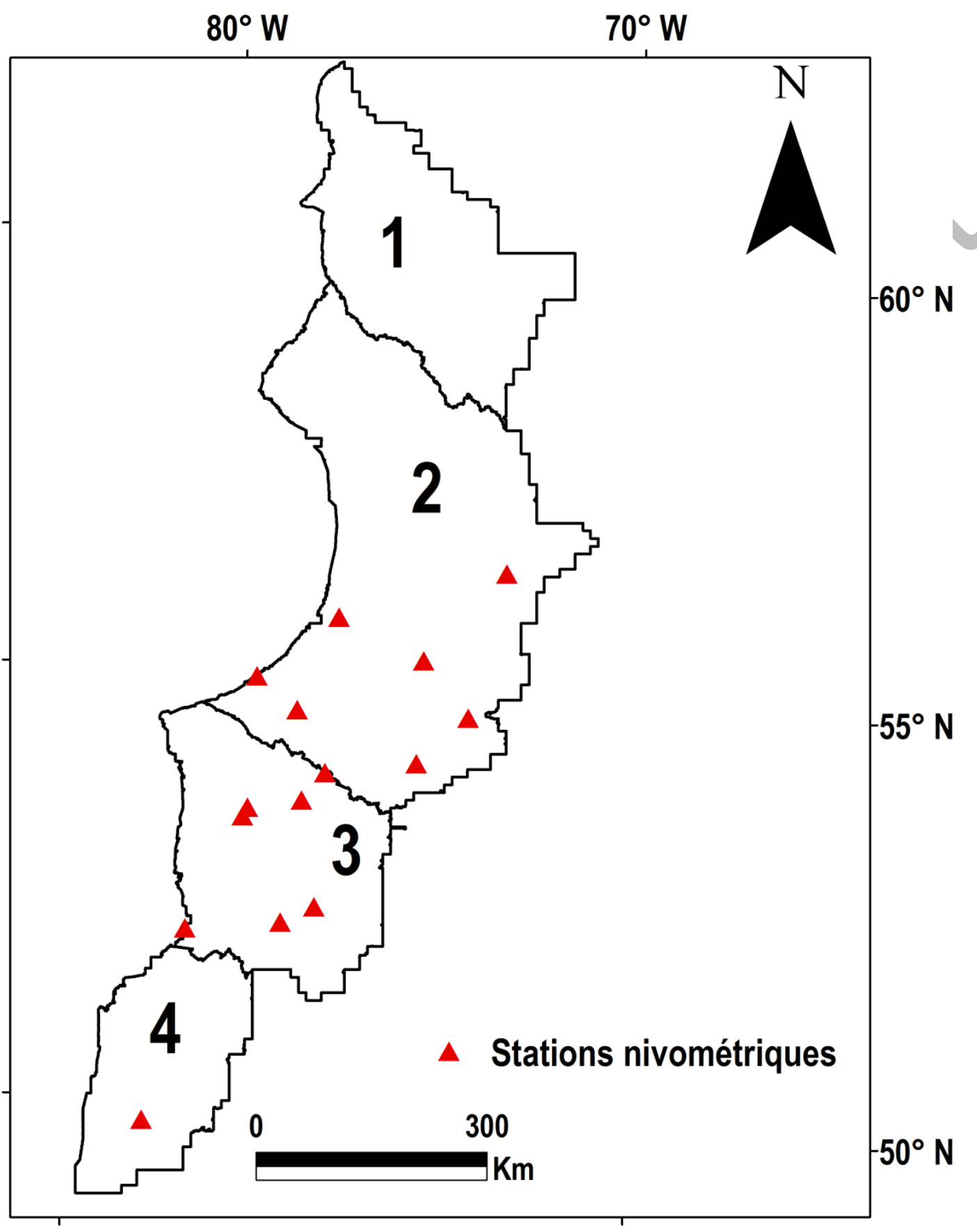




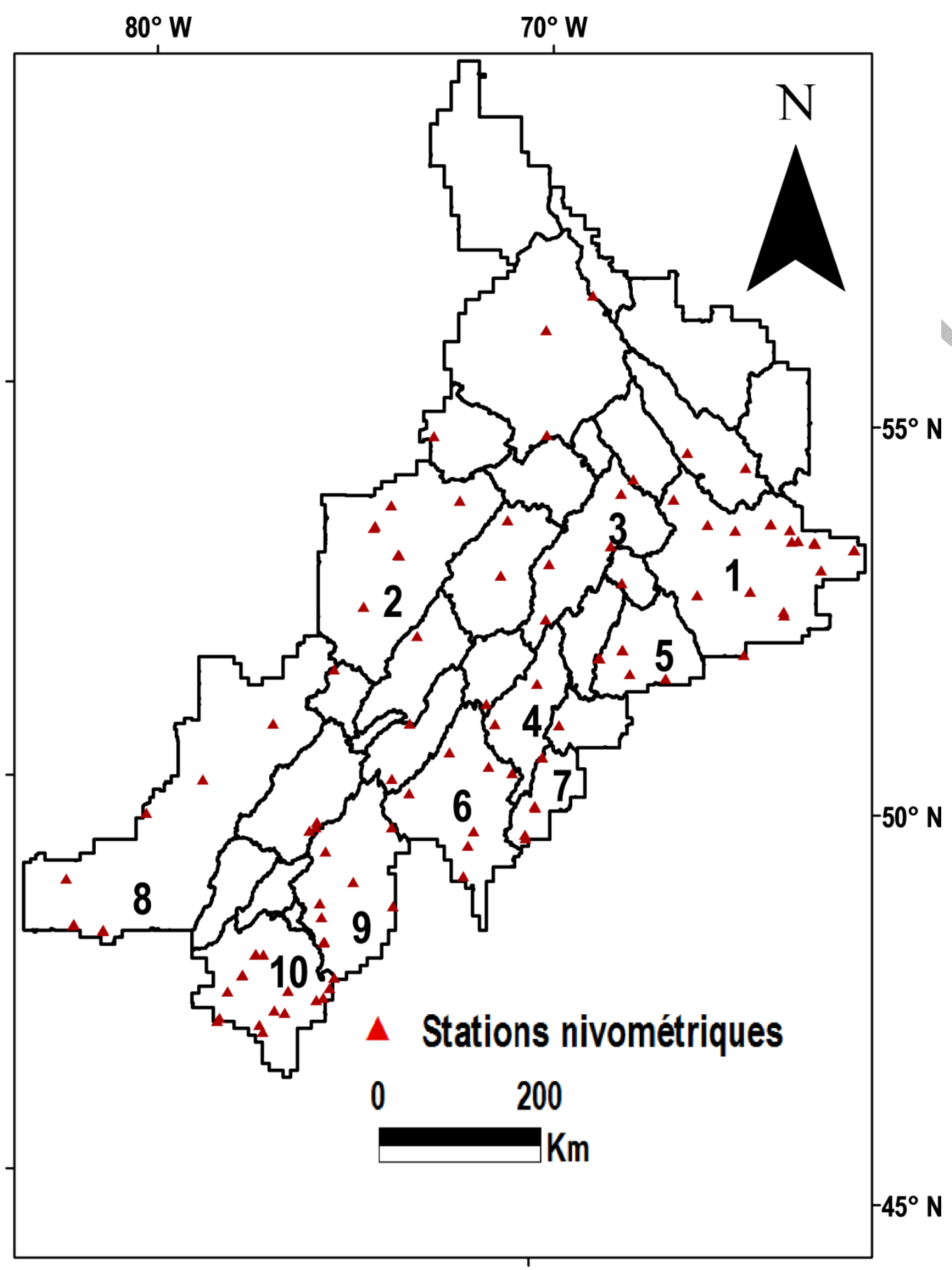




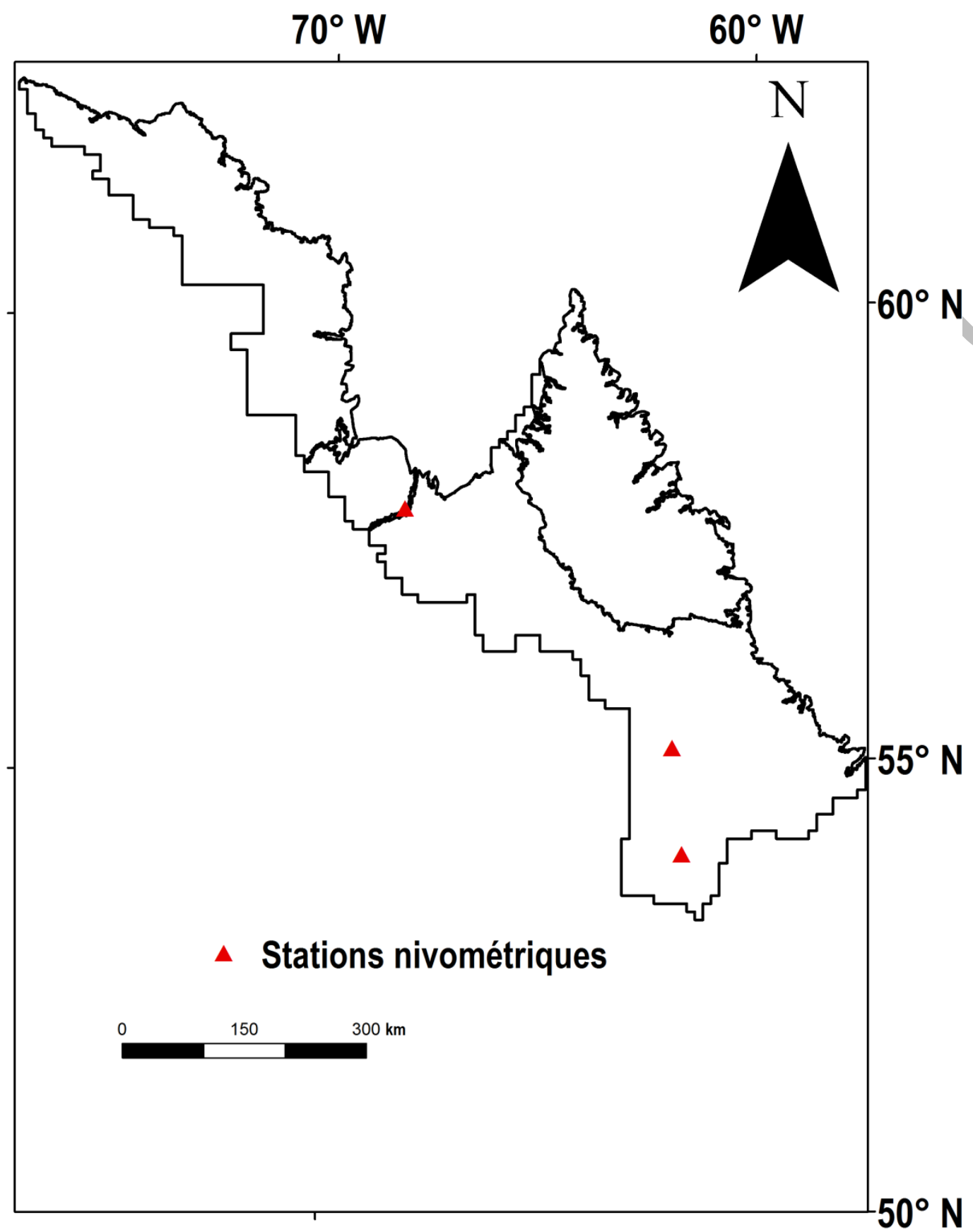




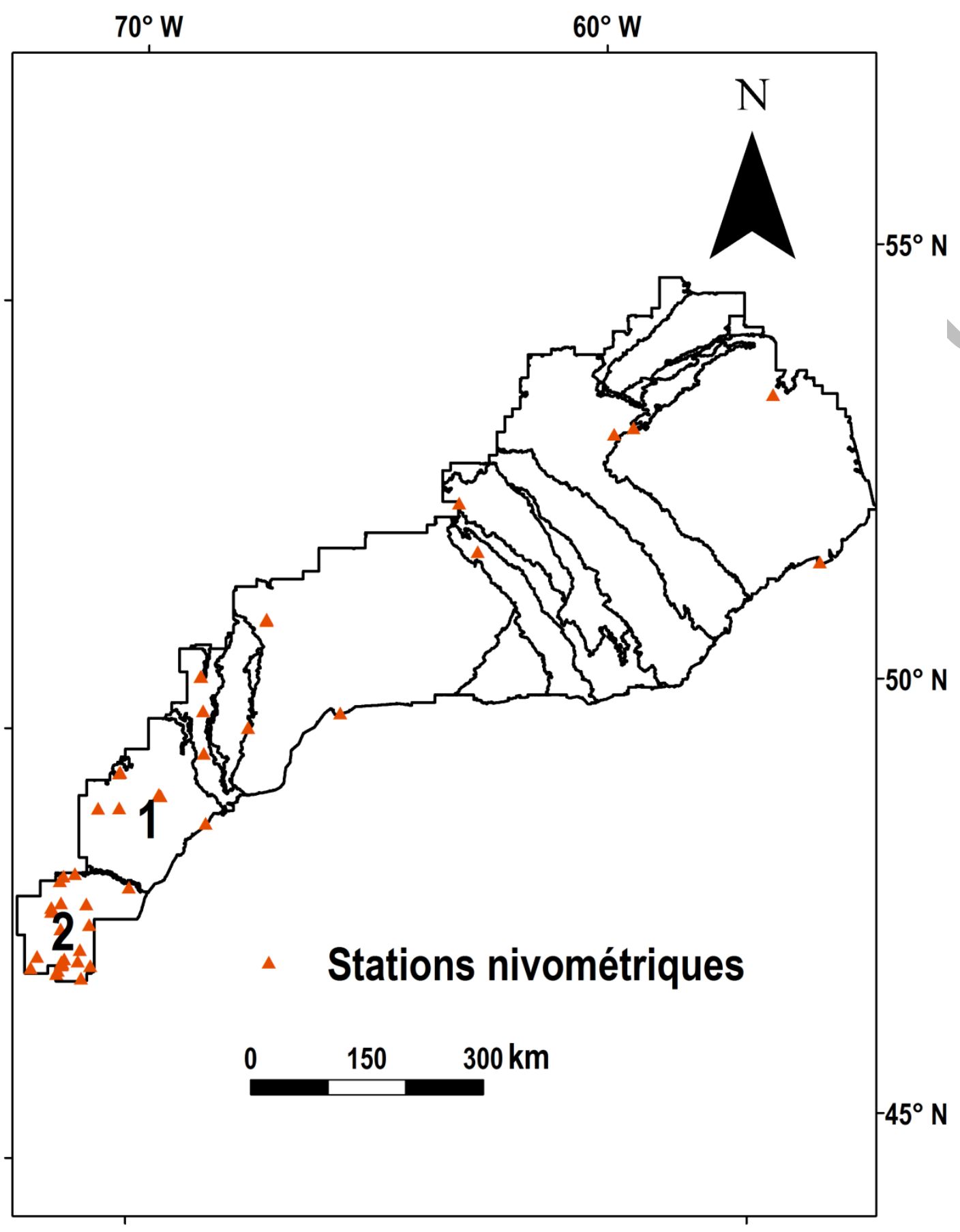




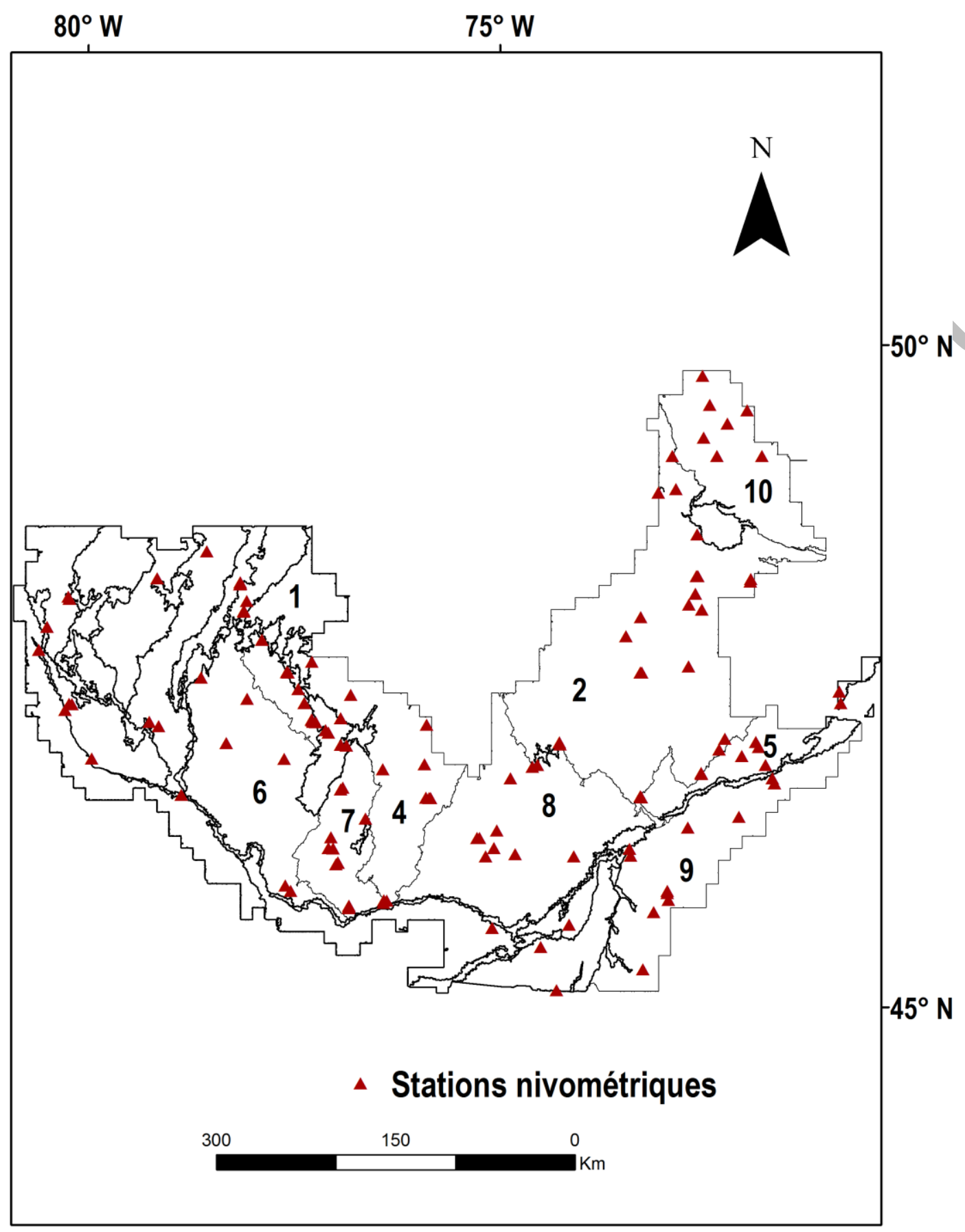




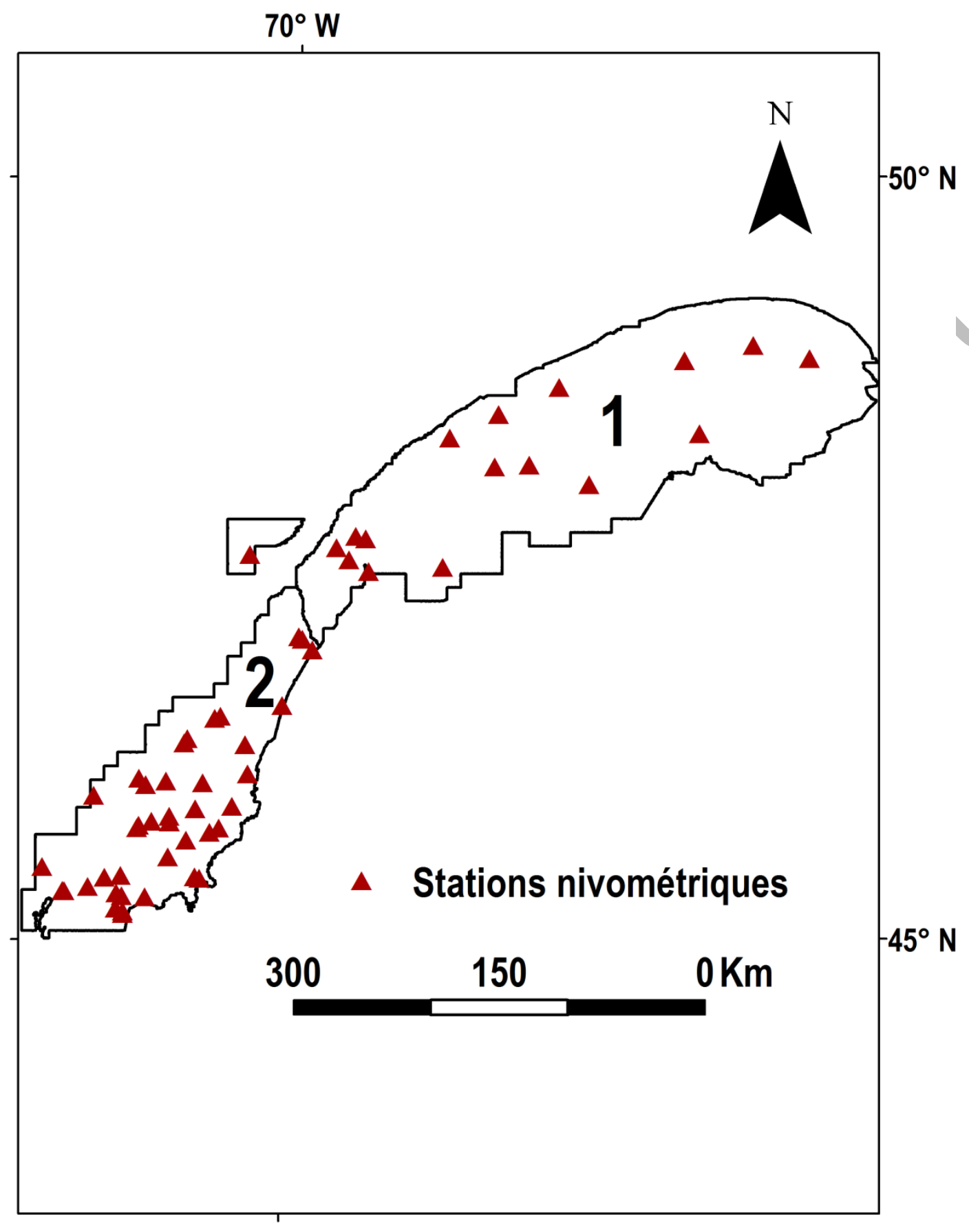


Table 1 Comparaison entre les segments contigus à l'échelle régionale ( $p$ du test de Kruskal-Wallis et les box-plots);

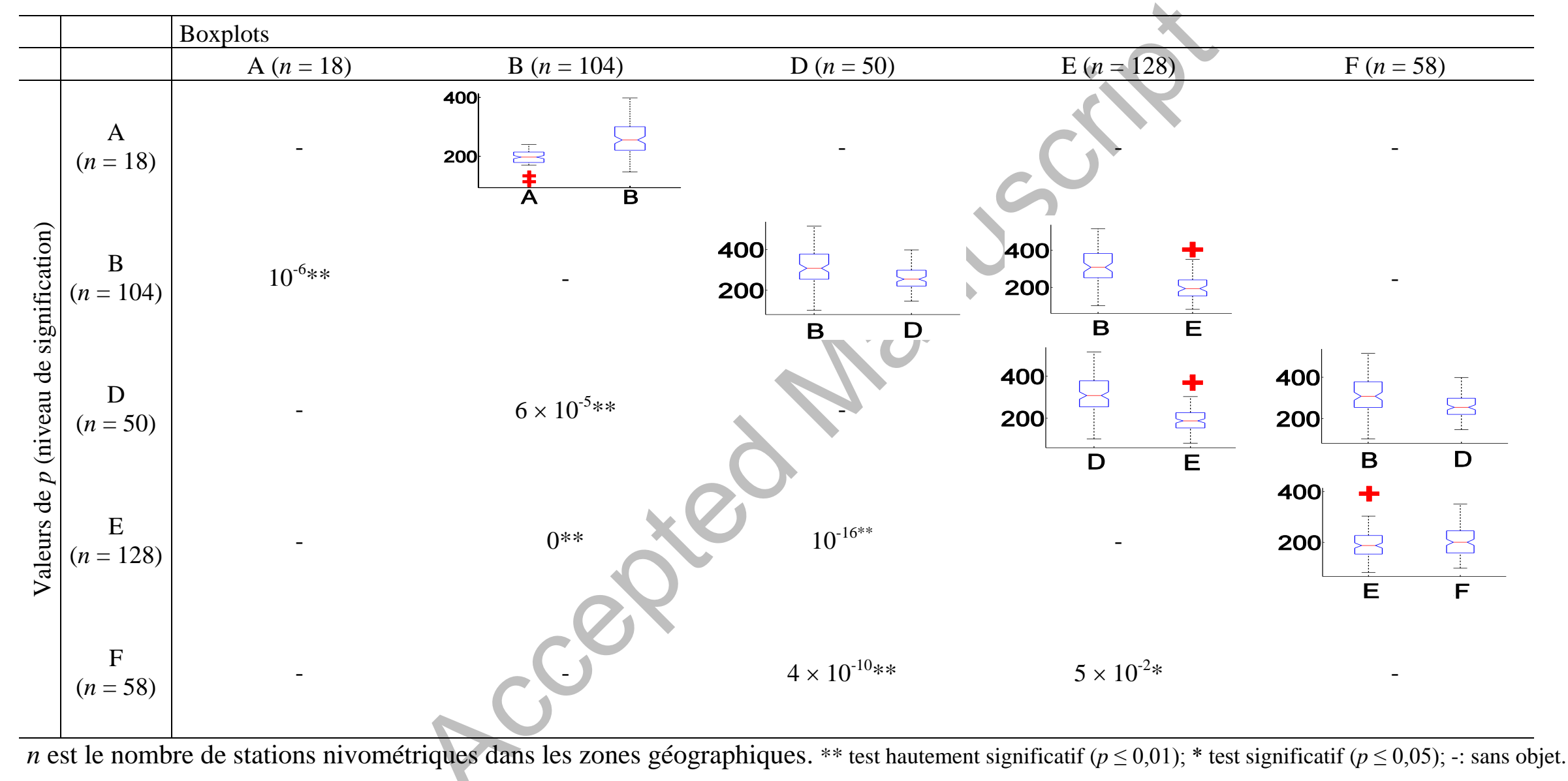


Table 2 Comparaison entre les segments contigus de la zone A à l'échelle locale (Les pvalues du test de Kruskal-Wallis et les box-plots).

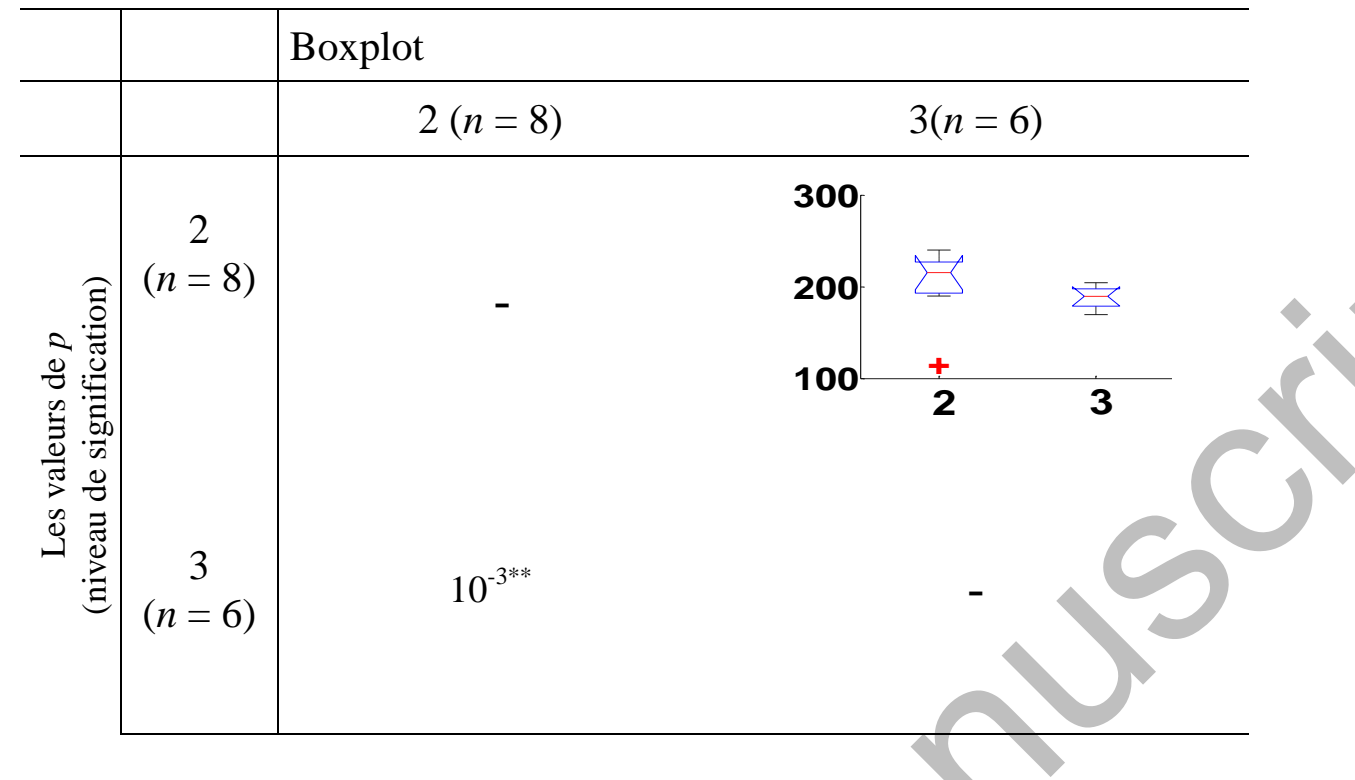

-: sans objet; $n=$ nombre de stations nivométriques dans les zones géographiques **: Test significatif (Les valeurs de $p \leq 0,01$ ) 
Table 3 Comparaison entre les segments contigus de la zone B à l'échelle locale (Les p-values du test de Kruskal-Wallis et les box-plots).

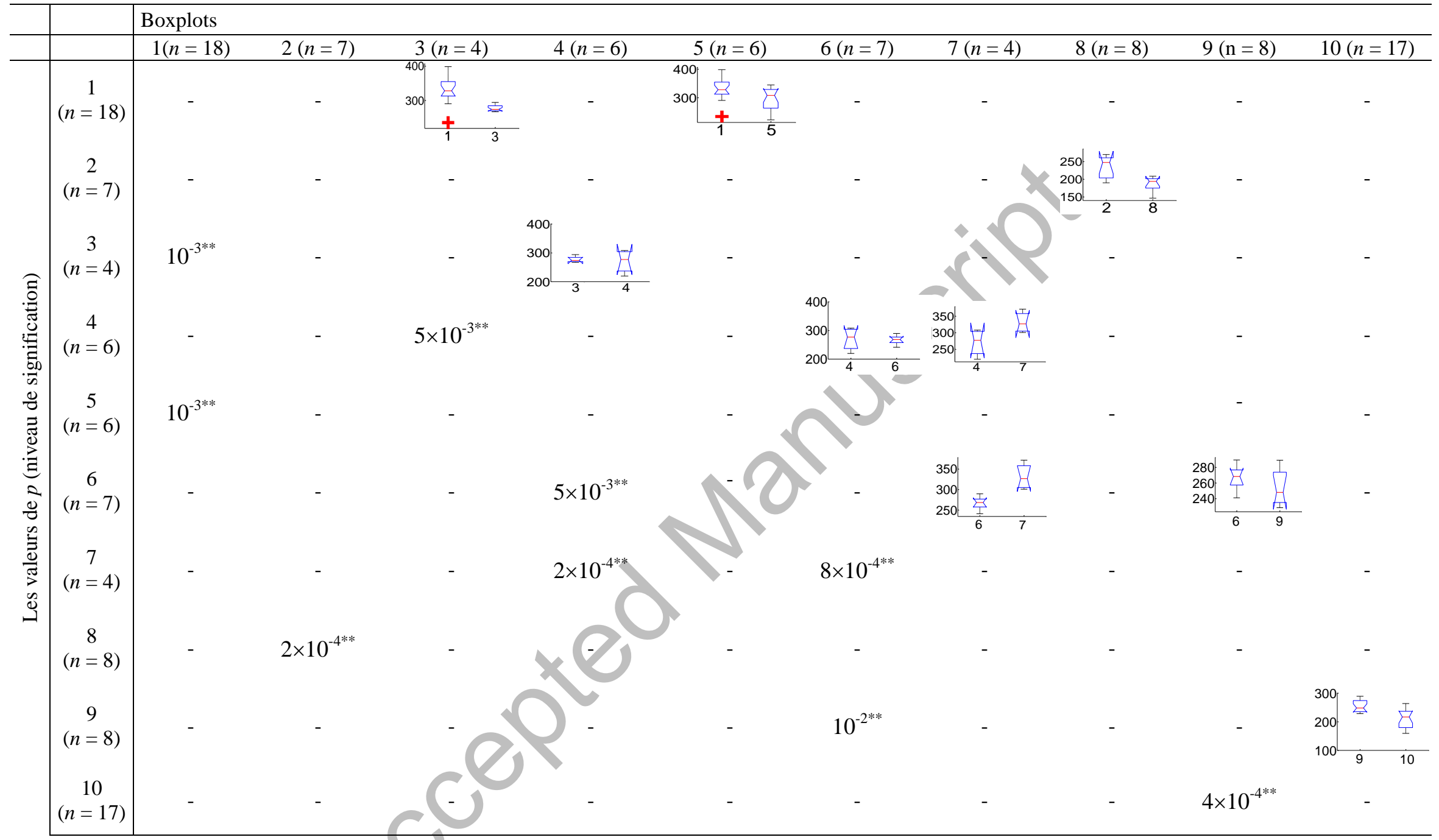

-: sans objet; $n=$ nombre de stations nivométriques dans les zones géographiques ; **: Test hautement significatif (p-value $\leq 0,01)$;

$*$ : Test significatif ( $\mathrm{p}$-value $\leq 0,05$ ) 
Table 4 Comparaison entre les segments contigus de la zone D à l'échelle locale (Les p- values du test de Kruskal-Wallis et les box-plots).

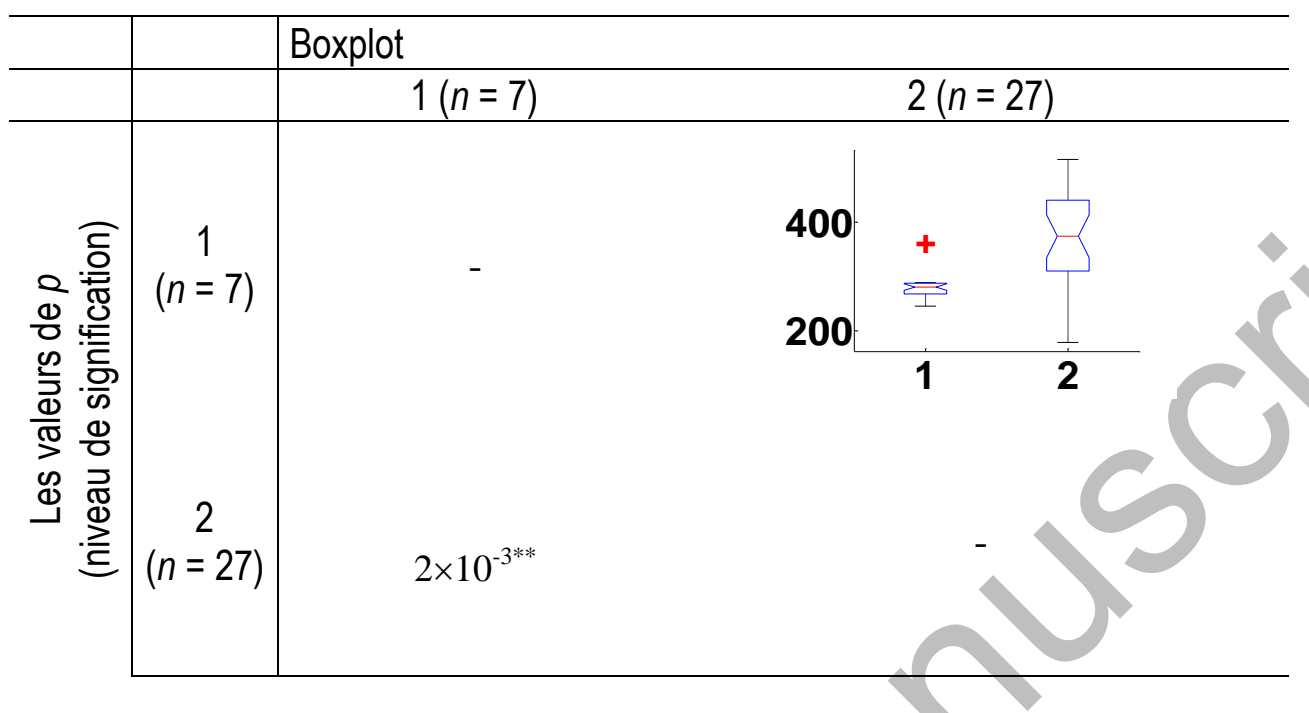

-: sans objet; $n=$ nombre de stations nivométriques dans les zones géographiques **: Test hautement significatif (Les valeurs de $p \leq 0,01$ ) 
Table 5 Comparaison entre les segments contigus de la zone E à l'échelle locale (Les p-values du test de Kruskal-Wallis et les box-plots).

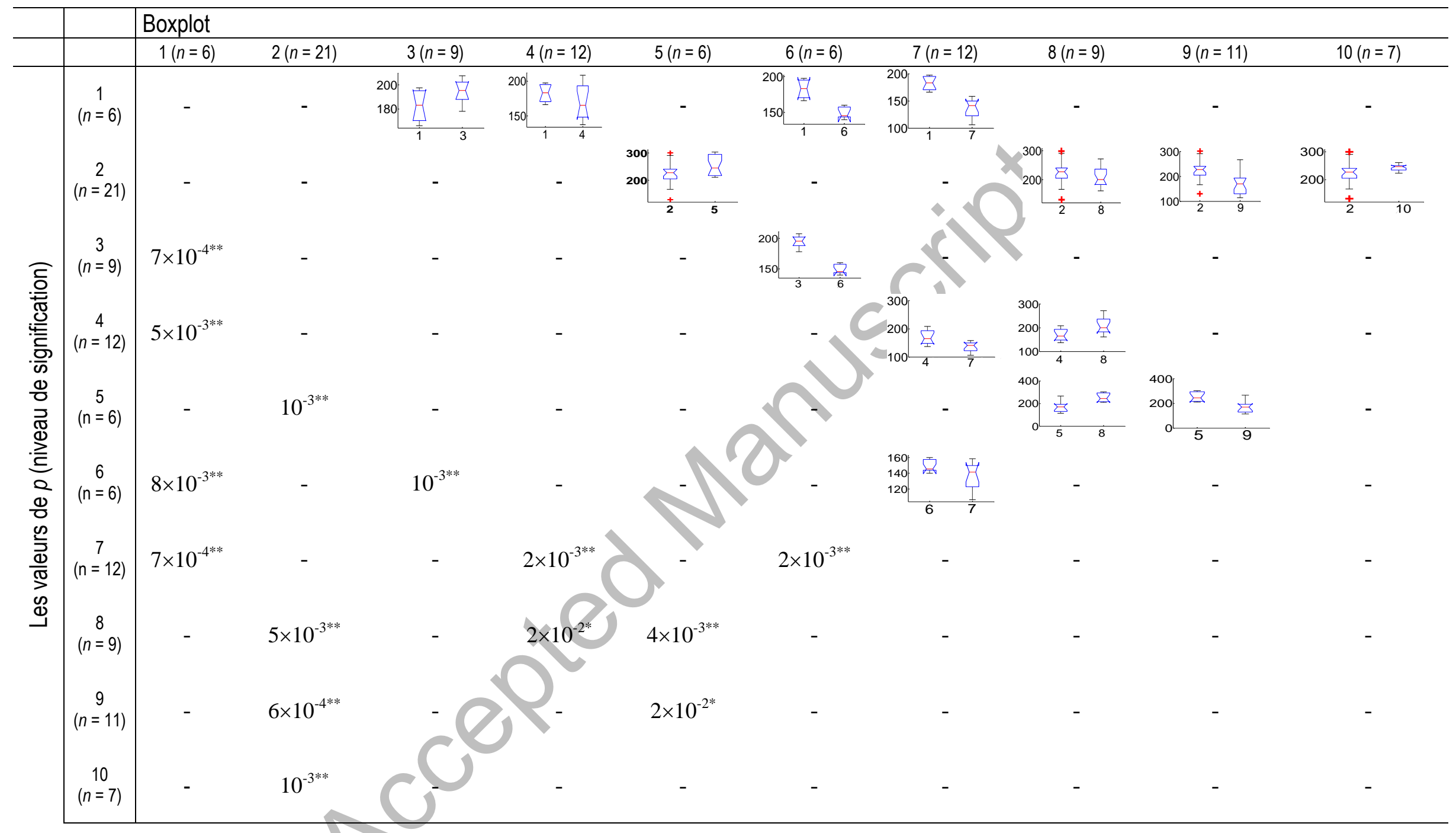

-: sans objet; $\mathrm{n}=$ nombre de stations nivométriques dans les zones géographiques; $* *$ : Test hautement significatif (Les valeurs de $p \leq 0,01$ ) ; *: Test significatif (Les valeurs de $p \leq 0,05$ ) 
Table 6 Comparaison entre les segments contigus de la zone F à l'échelle locale (Les valeurs de $p$ du test de Kruskal-Wallis et les boxplots)

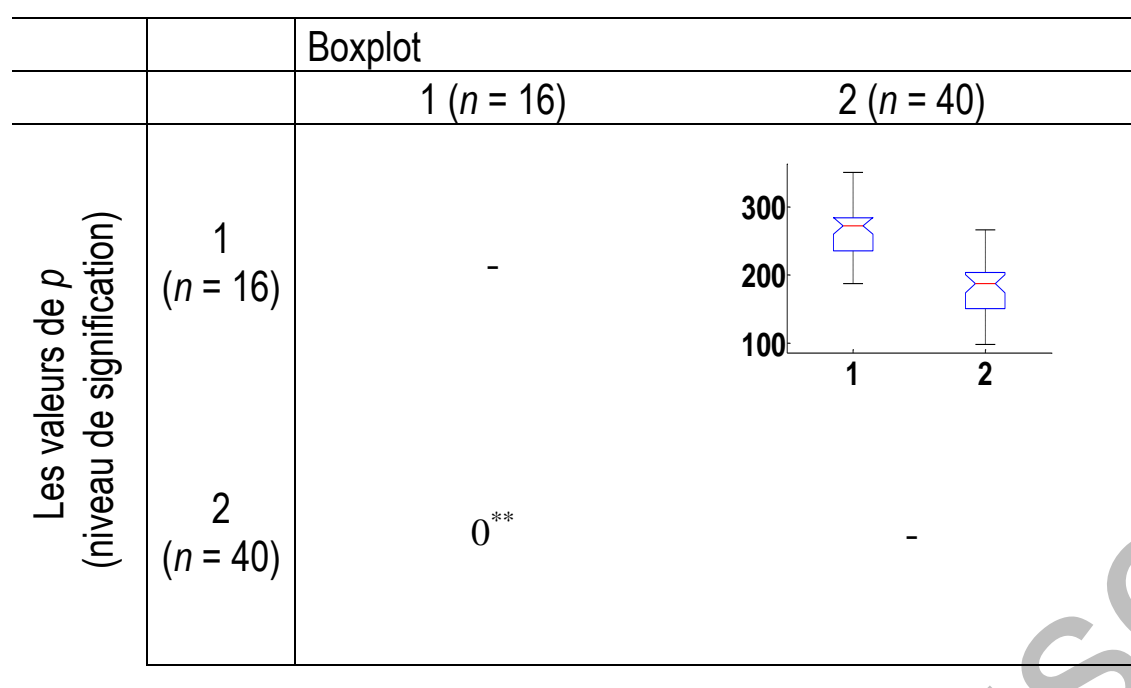

-: sans objet; $n=$ nombre de stations nivométriques dans les zones géographiques

$* *$ : Test hautement significatif (les valeurs de $p \leq 0,01$ ) 